\title{
A multidisciplinary approach unravels early and persistent effects of X-ray exposure at the onset of prenatal neurogenesis
}

Tine Verreet ${ }^{1,2}$, Roel Quintens ${ }^{1}$, Debby Van Dam ${ }^{3}$, Mieke Verslegers ${ }^{1}$, Mirella Tanori ${ }^{4}$, Arianna Casciati ${ }^{4}$, Mieke Neefs' ${ }^{1}$, Liselotte Leysen ${ }^{1}$, Arlette Michaux ${ }^{1}$, Ann Janssen ${ }^{1}$, Emiliano D’Agostino ${ }^{5}$, Greetje Vande Velde ${ }^{6,7}$, Sarah Baatout ${ }^{1}$, Lieve Moons ${ }^{2}$, Simonetta Pazzaglia ${ }^{4}$, Anna Saran ${ }^{4}$, Uwe Himmelreich ${ }^{6,7}$, Peter Paul De Deyn ${ }^{3,8}$ and Mohammed Abderrafi Benotmane ${ }^{1^{*}}$

\begin{abstract}
Background: In humans, in utero exposure to ionising radiation results in an increased prevalence of neurological aberrations, such as small head size, mental retardation and decreased IQ levels. Yet, the association between early damaging events and long-term neuronal anomalies remains largely elusive.

Methods: Mice were exposed to different X-ray doses, ranging between 0.0 and 1.0 Gy, at embryonic days (E) 10, 11 or 12 and subjected to behavioural tests at 12 weeks of age. Underlying mechanisms of irradiation at E11 were further unravelled using magnetic resonance imaging (MRI) and spectroscopy, diffusion tensor imaging, gene expression profiling, histology and immunohistochemistry.
\end{abstract}

Results: Irradiation at the onset of neurogenesis elicited behavioural changes in young adult mice, dependent on the timing of exposure. As locomotor behaviour and hippocampal-dependent spatial learning and memory were most particularly affected after irradiation at E11 with $1.0 \mathrm{~Gy}$, this condition was used for further mechanistic analyses, focusing on the cerebral cortex and hippocampus. A classical p53-mediated apoptotic response was found shortly after exposure. Strikingly, in the neocortex, the majority of apoptotic and microglial cells were residing in the outer layer at $24 \mathrm{~h}$ after irradiation, suggesting cell death occurrence in differentiating neurons rather than proliferating cells. Furthermore, total brain volume, cortical thickness and ventricle size were decreased in the irradiated embryos. At 40 weeks of age, MRI showed that the ventricles were enlarged whereas $\mathrm{N}$-acetyl aspartate concentrations and functional anisotropy were reduced in the cortex of the irradiated animals, indicating a decrease in neuronal cell number and persistent neuroinflammation. Finally, in the hippocampus, we revealed a reduction in general neurogenic proliferation and in the amount of Sox2-positive precursors after radiation exposure, although only at a juvenile age.

Conclusions: Our findings provide evidence for a radiation-induced disruption of mouse brain development, resulting in behavioural differences. We propose that alterations in cortical morphology and juvenile hippocampal neurogenesis might both contribute to the observed aberrant behaviour. Furthermore, our results challenge the generally assumed view of a higher radiosensitivity in dividing cells. Overall, this study offers new insights into irradiation-dependent effects in the embryonic brain, of relevance for the neurodevelopmental and radiobiological field.

Keywords: Apoptosis, Brain development, Cognitive dysfunction, MRI, Radiation

\footnotetext{
* Correspondence: rafi.benotmane@sckcen.be

'Radiobiology Unit, Laboratory of Molecular and Cellular Biology, Institute for Environment, Health and Safety, Belgian Nuclear Research Centre, SCK.CEN, $2400 \mathrm{Mol}$, Belgium

Full list of author information is available at the end of the article
} 


\section{Background}

Central nervous system development is a protracted process comprising a dynamic equilibrium between proliferation, differentiation, migration, synaptogenesis, myelination and cell death [1]. Disturbance of any of these events, both pre- or postnatally, might have serious consequences for adult brain structure and function [2]. Ionising radiation is, amongst other stressors like ethanol, drugs and infectious agents [3], known to cause such a perturbation of brain development, although effects are strongly dependent on the timing and dosage of exposure [4]. Knowledge about the long-term impact of prenatal irradiation has been largely based on epidemiological studies of atomic bomb survivors, in which children in utero exposed to radiation had a higher incidence of severe mental retardation and growth impairment [5]. In addition, a decrease in intelligence and school performance and an increased risk for seizures were noted [6]. Since pregnant women can be exposed to radiation during medical examination [7] and/or treatment, particularly during radiotherapy [8], a high concern and the clear need for increased knowledge exists concerning the long-term outcome of the exposed embryo. The risk for a mother to develop cancer during pregnancy is rare (about one in 1,000 to 2,000 pregnancies), but when it occurs, radiotherapy can be considered as treatment during the first and second trimesters. However, the dose to the embryo should be kept as low as reasonably achievable [9]. Successful radiotherapy treatment of pregnant women with breast cancer and Hodgkin's disease has been reported, with the birth of healthy children [8]. However, it should be noted that so far, these in utero exposed patients have been followed up only until childhood. Therefore, the possibility of late side effects, such as subclinical cognitive problems, cannot be excluded.

Epidemiological evidence further suggests that radiationinduced long-term neuronal effects only arise when the exposure occurred between weeks 8 and 15 of pregnancy and to a lesser extent between weeks 16 and 25 [5,6]. The timing of irradiation thus seems decisive for the manifestation of late effects. The period of weeks 8 to 15 of human gestation is characterised by a fast increase in neuronal cell number, as well as the start of neuronal differentiation and the consequent migration to the cortical plate [10]. Of note, during this developmental period, only a limited migration and final cell positioning takes place. Indeed, by the 20th week of gestation, the cortical plate consists of less than $6 \times 10^{9}$ cells [11], which only accounts for approximately $25 \%$ of the adult neocortical cell number [12]. This thus implies that the remaining $75 \%$ of neurons still need to migrate to their final destination in the cortex. In the mouse, the radiosensitive period of neurogenesis roughly takes place between embryonic days (E) 12 and E16 [13], whereas the onset of murine neurogenesis occurs at E10 [2,14], shortly after neural tube closure [15]. In animal models, irradiation at other developmental time points than those corresponding to weeks 8 to 15 in humans have also been studied [16,17]. In contradiction to epidemiological evidence, they indicate the possibility of neurological deficits outside the most radiosensitive developmental period. For example, in non-human primates, radiation exposure before neurogenesis but after neural tube closure has been shown to result in adult-onset cognitive impairment [18]. Interestingly, in utero brain development in rhesus monkeys occurs slow when compared to rodents, allowing irradiation after neural tube closure, but still before the onset of neurogenesis [19]. This specific developmental time window cannot be investigated in mice, as neural tube closure coincides with the start of neurogenesis [20]. Thus, although evidence indicates that irradiation at the onset of neurogenesis may also induce long-term effects, at present this has not yet been studied in a rodent model.

Therefore, as an attempt to study radiosensitivity outside of the currently proposed radiosensitive period in a mouse model, we irradiated animals at the start of neurogenesis, i.e. at E10, E11 or E12 and we performed a detailed investigation to delineate short- and long-term radiation injury to the brain. In a first instance, we assessed radiation effects by subjecting the offspring to a behavioural test battery at the age of 12 weeks. This showed that behavioural changes due to radiation exposure were timing-specific. Irradiation at E10 resulted in an impaired consolidation of reference memory without spatial learning effects, whereas animals exposed at E11 showed both spatial learning and long-term memory deficits. When the irradiation occurred at E12, no changes in learning and memory could be observed. Therefore, we performed a multidisciplinary study in order to unravel early and persistent radiation-induced mechanisms, with a focus on mice irradiated at E11. Hereto, we combined multiple in vivo magnetic resonance imaging (MRI) paradigms, as well as gene expression analyses and immunohistochemistry, specifically focusing on the cerebral cortex and hippocampus. With this study, we establish a possible link between early radiation-induced effects and the longterm functional and morphological outcome.

\section{Methods}

\section{Animals and irradiation}

All animal experiments were performed in accordance with the European Communities Council Directive of November 24, $1986(86 / 609 /$ EEC) and approved by the local ethical SCK•CEN/VITO (ref. 02-012), University of Antwerp and KU Leuven committees. C57Bl/6 J were purchased from Janvier (Bio Services, Uden, The Netherlands) and housed under standard laboratory conditions (12-h light/dark cycle). Food and water were available ad libitum. 
Female mice were coupled during a 2 -h time period in the morning, at the start of the light phase $(7.30 \mathrm{~h}$ until $9.30 \mathrm{~h}$ ), in order to ensure synchronous timing of embryonic development. Subsequently, pregnant females were whole-body-irradiated at E10, 11 or $12(0.1,0.2,0.5$ or $1.0 \mathrm{~Gy})$ at a dose rate of $0.35 \mathrm{~Gy} \mathrm{~min}^{-1}$ using a Pantak RX tube operating at $250 \mathrm{kV}$ and $15 \mathrm{~mA}$ (1-mm Cu-filtered $\mathrm{X}$-rays). The calibration of the $\mathrm{X}$-ray tube was performed using an ionisation chamber. Control mice were shamirradiated. The offspring was used in further experiments.

\section{Behavioural tests}

At the age of 12 weeks, male animals (irradiated at E10, 11 or 12 with a dose of $0.0,0.2,0.5$ or $1.0 \mathrm{~Gy}$ ) were used in behavioural tests. At least eight animals were used in each test (except for the 1.0-Gy irradiated group at E12 in the Morris water maze (MWM) and cage activity $(N=4)$ and for the 0.5 -Gy irradiated group at E12 in the rotarod $(N=4))$.

\section{MWM learning and memory}

The MWM setting consisted of a circular pool (diameter: $150 \mathrm{~cm}$, height: $30 \mathrm{~cm}$ ) surrounded by invariable visual extramaze cues. The water was opacified with white non-toxic paint and kept at $25^{\circ} \mathrm{C}$. A round acrylic glass platform (diameter: $15 \mathrm{~cm}$ ) was placed $1 \mathrm{~cm}$ below the water surface at a fixed position. The acquisition or training phase comprised eight trial blocks of four daily trials starting from four different positions around the border of the maze in a semi-random order with a 15-min inter-trial interval. In case an animal was unable to reach the platform within $120 \mathrm{~s}$, it was placed on the platform for $15 \mathrm{~s}$ before being returned to its home cage. Animals' trajectories were recorded using a computerised videotracking system (EthoVision, Noldus, The Netherlands) logging escape latency. Four days after the final acquisition trial block, a probe trial was performed. For this purpose, the platform was removed from the maze and each mouse was allowed to swim freely for $100 \mathrm{~s}$. Spatial acuity was expressed as the percentage of time spent in the four different quadrants of the MWM.

\section{Cage activity recording}

Ambulatory cage activity was measured in solitary housed animals using standard transparent mouse cages $(22.5 \times$ $16.7 \times 14 \mathrm{~cm}$, length $\times$ width $\times$ height) placed between three infrared beams (two perpendicular to and one parallel with the length of the cage). Sensors were positioned $3 \mathrm{~cm}$ above the nesting material and detected horizontal activity. Cages were placed in closed cabinets accommodated with electricity-driven ventilation fans to keep optimum temperature and lights to imitate the animals' 12-h light/dark cycle. The number of beam interruptions in a 23-h period was counted using a microprocessor counter linked to a computer running in-house developed software. Recording started at $16 \mathrm{~h}$ and ended at $15 \mathrm{~h}$. Counts were summed over subsequent 30 -min periods.

\section{Accelerating rotarod}

Motor coordination and equilibrium were tested on an accelerating rotarod apparatus (Ugo Basile, Italy). After two adaptation trials of a maximum of 2 min each at a constant speed of $4 \mathrm{rpm}$, a mouse was placed on the rotating rod for four test trials with an inter-trial interval of $1 \mathrm{~min}$. The time (latency) a mouse could hold itself on the rod was measured up to a maximum of $5 \mathrm{~min}$, during which the rotation speed gradually increased from 4 to $40 \mathrm{rpm}$.

\section{Statistics}

Statistical analyses were performed using SigmaStat software (SPSS Inc., Erkrath, Germany). We applied a twoway ANOVA with treatment and quadrant as sources of variation to assess spatial memory in the MWM probe trial. For tests repeated over time (e.g. MWM acquisition), we used a two-way repeated measures (RM) ANOVA. Into more detail, two-way RM ANOVA was used to evaluate the significance of differences between latency scores during acquisition trial blocks in the MWM. Treatment group and trial block were considered as possible sources of variation. In case of a significant $P$ value, a post hoc Tukey multiple comparison test was performed. For cage activity, we examined general differences in activity patterns using two-way RM ANOVA with treatment and time (i.e. subsequent summed 30-min activity counts) as sources of variation. Rotarod latency was assessed using two-way RM ANOVA with irradiation dose and trial as possible sources of variation. If required, Tukey multiple comparison was applied. Statistical analyses were performed at a significance level of 0.05 .

\section{MR experiments}

At 1, 4, 12, 20 and/or 40 weeks after birth, in utero irradiated female and male mice $(0.0(N=5)$ or $1.0 \mathrm{~Gy}(N=6)$ at E11) were imaged using in vivo MRI. For these MR experiments, anaesthesia was induced in an induction chamber with $3-4 \%$ isoflurane in $100 \%$ oxygen. In the MR scanner, the anaesthesia was maintained at 1-2\% isoflurane in $100 \%$ oxygen. Respiration and body temperature were monitored throughout the measurements and maintained at 70-90 $\mathrm{min}^{-1}$ and $37^{\circ} \mathrm{C}$, respectively. MR images and spectra were acquired using a 9.4-T Biospec smallanimal MR scanner (Bruker BioSpin, Ettlingen, Germany) with a horizontal bore of $20 \mathrm{~cm}$ and equipped with actively shielded gradients $\left(600 \mathrm{mT} \mathrm{m}^{-1}\right)$. We also used a 7.2-cm linearly polarised resonator for transmission and an actively decoupled, circular polarised mouse head surface coil for receiving (both Bruker BioSpin). After 
acquisition of 2D multi-slice localizer images, 3D T2weighted MRI, diffusion tensor imaging (DTI) and singlevolume MR spectroscopy (MRS) were performed. In order to reduce scanning time, and therefore extended periods of anaesthesia that might affect brain development, DTI measurements were performed only at 12 and 40 weeks after birth and MRS at week 40 .

\section{D T2-weighted MRI}

A rapid acquisition with refocused echoes (RARE) protocol was acquired to obtain 3D T2-weighted MR images using the following parameters: echo time $(\mathrm{TE})=12 \mathrm{~ms}$, repetition time $(\mathrm{TR})=1,000 \mathrm{~ms}$, RARE factor $=10$, field of view $(\mathrm{FOV})=2.0 \times 1.28 \times 1.28 \mathrm{~cm}^{3}$, matrix $=200 \times 128 \times 128$, isotropic resolution $=100 \mu \mathrm{m}$, number of averages $=1$. For repeated analysis of the brain structures (motor cortex and ventricles), we identified these structures on every 3D T2-weighted MR image (axial view) based on the brain atlas of Franklin and Paxinos [21]. Confirmation was based on the MRI-based brain atlas provided by the National University of Singapore (www.bioeng.nus.edu.sg/cfa/ mouse_atlas.html) [22].

The thickness of the motor cortex was manually measured in the axial slice in which lateral ventricles and the dorsal third ventricle meet (illustrated in Additional file 1: Figure S1A). The distance between the corpus callosum (hypointense structure) and the boundary of the brain was measured.

The volume of the ventricles was semi-manually delineated using the region of interest tool of the ParaVision software (Bruker BioSpin). A seeding point was defined in the hyperintense ventricles (Additional file 1: Figure S1B). The automatic delineation of the hyperintense ventricles was manually corrected.

\section{DTI}

DTI data were acquired using an echo planar imaging (EPI) sequence with spin echo read-out. The following parameters were used: $\mathrm{TE}=32 \mathrm{~ms}, \mathrm{TR}=5,000 \mathrm{~ms}, b$ values $=0.125 \mathrm{~s} \mathrm{~mm}^{-2}$, number of gradient encoding directions $=21$, 20 slices (no gap) of $0.5-\mathrm{mm}$ thickness, FOV $=2.6 \times 1.9 \mathrm{~cm}^{-2}$, matrix $254 \times 192$, in-plane resolution $=100 \mu \mathrm{m}$, number of averages $=10$. Fractional anisotropy (FA) values were determined using the DTI reconstruction tool of the ParaVision 5.1 software (Bruker BioSpin). Regional FA was obtained by placing spherical regions of interest in the respective brain regions using the ParaVision software. Brain regions were identified in the 3D T2-weighted MR images and transferred to the DTI data set that was acquired during the same anaesthesia session. The brain regions studied were the corpus callosum (1), sensory-motor cortex (2), hippocampus (3) and thalamus (4) (Additional file 1: Figure S1C).

\section{MRS}

Single-voxel $1 \mathrm{H}$ MRS was performed as described previously [23,24]. In brief, the PRESS pulse sequence with implemented pre-delay outer volume suppression, as well as the water suppression method VAPOR, were used. MRS parameters were as follows: $\mathrm{TR}=2 \mathrm{~s}, \mathrm{TE}=$ $20 \mathrm{~ms}$, spectral width $=4 \mathrm{kHz}$, number of averages $=256$ to 400. Volumes of interest (voxels) were placed in the region of the cortex $\left(1.3 \times 1.3 \times 2.8 \mathrm{~mm}^{3}\right)(1)$ and striatum $\left(1.7 \times 2.4 \times 1.4 \mathrm{~mm}^{3}\right)$ (2) (as illustrated in Figure $1 \mathrm{~A}$ ). Spectra were corrected for B0 instability due to Eddy currents and B0 drift using the Bruker built-in routines. Shimming was performed using FASTMAP for initial shimming and subsequently by manual shimming on the selected volumes, which resulted in a final water line width of $<25 \mathrm{~Hz}$. MR spectra were further processed using the jMRUI software [23,24]. This analysis included filtering out the residual water signal, phase correction and baseline correction. Signal quantification was performed using AQSES by fitting a linear combination of metabolite profiles to the experimental data and by modelling of the baseline using splines [25]. The basis set of metabolites included alanine, aspartate, creatine and phosphocreatine, $\gamma$-aminobutyrate, glucose, glutamine and glutamate, glycerophosphorylcholine and phosphorylcholine, lactate, myo-inositol, N-acetyl aspartate (NAA), phosphoryl ethanolamine and taurine. The unsuppressed water signal was acquired with identical parameters, except for the acquisition of only one average, a higher receiver gain and $\mathrm{TR}=10 \mathrm{~s}$. The unsuppressed water was used as an internal reference for the quantification of metabolites.

\section{Statistics}

Statistical analyses were performed using GraphPad Prism 5.0 (GraphPad Software, San Diego, CA, USA). Statistical significance was determined using two-way RM ANOVA for 3D T2-weighted MRI. ANOVA pairs of means were compared using the Bonferroni post hoc test. FA- and MRS-calculated concentration comparisons were performed using a two-tailed Student's $t$-test. $P$ values $<0.05$ were considered to be statistically significant.

\section{Gene expression analysis}

In order to identify early molecular mechanisms related to radiation exposure, embryonic brains were dissected and frozen for subsequent RNA extraction at 2 and $24 \mathrm{~h}$ after irradiation at E11 (0.0, 0.1, 0.2, 0.5 or $1.0 \mathrm{~Gy})$. For both microarrays and quantitative reverse transcriptase PCR (qRT-PCR), at least three embryos from different litters were used.

\section{Microarray preparation and analysis}

Total RNA was extracted from flash-frozen embryonic brains using the AllPrep DNA/RNA/Protein Mini Kit 


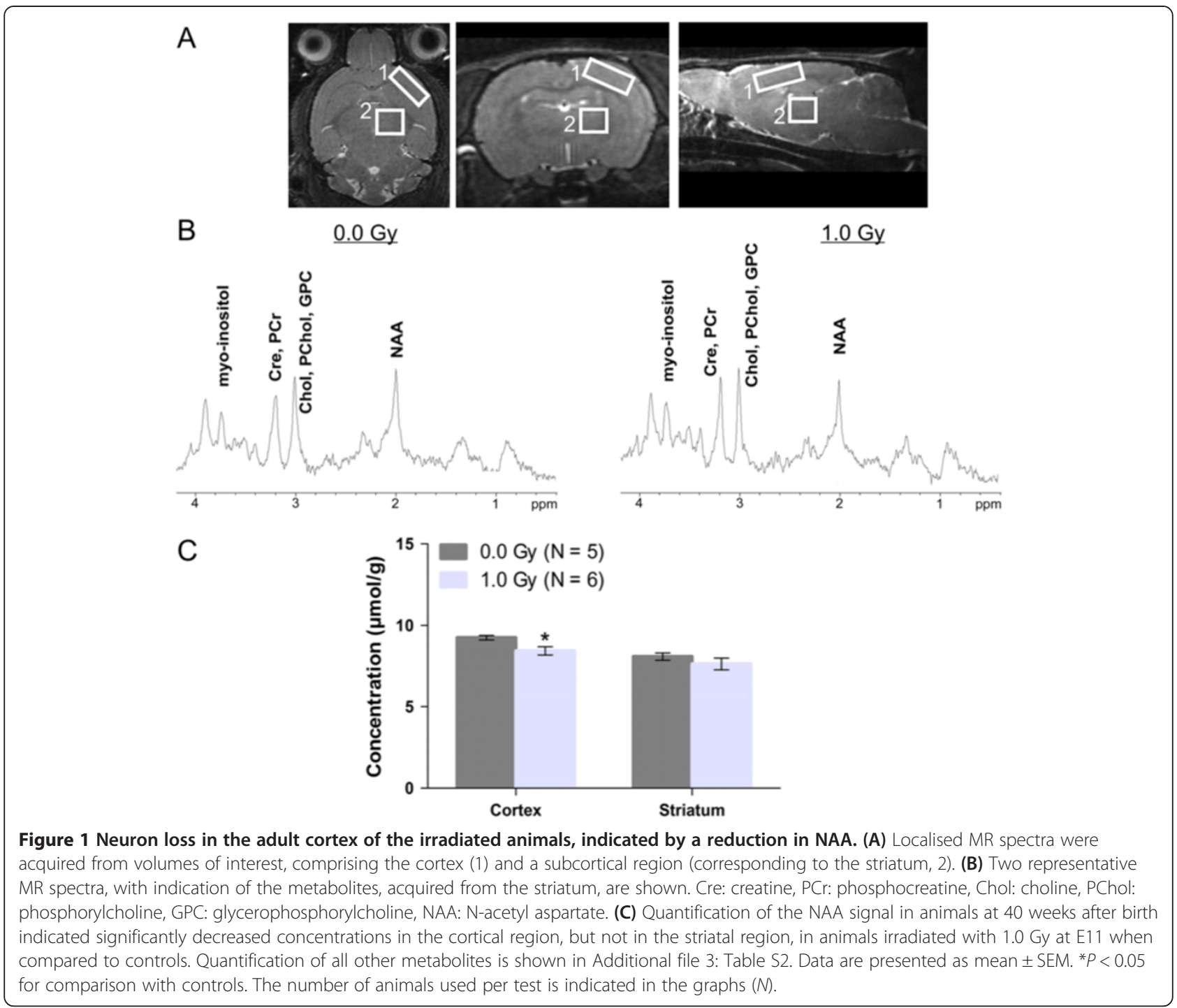

(Qiagen, Hilden, Germany) and quality-controlled using the 2100 BioAnalyzer (Agilent, Santa Clara, CA, USA). Only samples with a RNA integrity number $>8$ were used for hybridisation onto Affymetrix Mouse Gene 1.0 ST arrays (Affymetrix, Santa Clara, CA, USA) as per the manufacturer's recommendations. CEL files were uploaded to the Partek Genomics Suite (version 6.6). Data normalisation was performed using a customised Robust Multi-chip Analysis algorithm (background correction for entire probe sequence, quantile normalisation, $\log 2$ transformation of intensity signals). Microarray data are available in the ArrayExpress database (www.ebi.ac.uk/arrayexpress) under accession number E-MTAB-2720.

\section{Statistics}

To identify radiation-responsive genes, we first performed a filtering step, in which we excluded the $75 \%$ genes which showed the lowest variance across the different conditions. Next, we performed a two-way ANOVA with dose and scan date as possible sources of variation to account for differences due to possible batch effects. Significantly differentially expressed genes were identified as those with a $P$ value $<0.05$ after correction for multiple testing according to the method of Benjamini and Hochberg [26].

\section{Functional enrichment analysis}

For functional enrichment analysis, we used the GOrilla tool [27] with the following settings: Organism: Mus musculus, Running mode: Two unranked lists of genes (target list: differentially expressed genes, background list: genes expressed above background in at least 30\% of all samples, $P$ value threshold: 0.001$)$. The results of this analysis were subsequently reduced using REVIGO [28], with 
default settings. REVIGO serves to remove redundant Gene Ontology (GO) terms. The version of the GO used was as follows: go_201304-termdb.obo-xml.gz.

\section{Chromatin immunoprecipitation enrichment analysis}

Chromatin immunoprecipitation (ChIP) enrichment analysis [29] was performed to identify putative transcriptional regulators of differentially expressed genes. It combines published data from ChIP-chip, ChIP-seq, ChIP-PET and DamID experiments in order to rank transcription factors that are most likely responsible for observed gene expression changes based on statistical enrichment analysis. For this analysis, differentially expressed genes were used as input and databases from all species, cell types and ChIP methods were interrogated.

\section{qRT-PCR}

Complementary DNA was prepared from total RNA using the GoScript ${ }^{\mathrm{Tm}}$ Reverse Transcriptase kit (Promega, Leiden, The Netherlands) using $1 \mu \mathrm{l}$ of random hexamer primers and $3.75 \mathrm{mM} \mathrm{MgCl}_{2}$ in $20-\mu \mathrm{l}$ reactions. For quantitative PCR, we used the MESA GREEN kit (Eurogentec, Seraing, Belgium) according to the manufacturer's instructions. Briefly, duplicate $25-\mu \mathrm{l}$ reactions were performed using $200 \mathrm{nM}$ of forward and reverse primers. Reactions were run in an Applied Biosystems 7500 Fast Real-Time PCR instrument with an initial hold cycle of $5 \mathrm{~min}$ at $95^{\circ} \mathrm{C}$ followed by 40 cycles of denaturation for $3 \mathrm{~s}$ at $95^{\circ} \mathrm{C}$ and primer annealing/elongation for $45 \mathrm{~s}$ at $60^{\circ} \mathrm{C}$. Afterwards, a melting curve was performed to check for additional PCR products or primer dimers. For each of the tested primer pairs, we first assessed the reaction efficiencies by running standard curves from cDNA samples in which the respective genes were highly expressed. Reaction efficiencies were used for relative quantification using the method as described by Pfaffl [30]. Gapdh was used as an internal reference gene. Primers used for qRT-PCR are listed in Additional file 2: Table S1.

\section{Statistics}

To compare gene expression data obtained with qRTPCR, statistical analyses were performed using GraphPad Prism 5.0. Statistical significance was determined using a two-tailed Student's $t$-test for comparison between pairs of means. $P$ values $<0.05$ were considered to be statistically significant.

\section{Immunohistochemistry and morphometric analyses}

At $24 \mathrm{~h}$ and 1 week after irradiation $(0.0$ or $1.0 \mathrm{~Gy}$ at E11), pregnant mice were sacrificed by cervical dislocation and embryos were dissected and processed for immunohistochemical analysis. Juvenile animals (4 weeks old, 0.0 or $1.0 \mathrm{~Gy}$ at E11) were processed similarly to study hippocampal neurogenesis. At least three animals were used in each test.

\section{Tissue collection and staining}

Embryos and juvenile brains were fixed overnight in 10\% buffered formalin and dehydrated in a graded series of ethanol. Consequently, 4- $\mu \mathrm{m}$-thick paraffin sections were cut. Before incubation in $3 \% \mathrm{H}_{2} \mathrm{O}_{2}$ for $10 \mathrm{~min}$, sections were dewaxed, rehydrated and heated in unmasking buffer. The primary antibodies used included the following: polyclonal rabbit anti-cleaved caspase-3 (1/200, Cell Signaling Technology, Danvers, MA, USA), polyclonal rabbit anti-GFAP (1/500, Dako, Carpinteria, CA, USA), polyclonal rabbit anti-Sox2 (1/500, Abcam, Cambridge, UK), polyclonal rabbit anti-DCX (1/500, Abcam, Cambridge, UK), polyclonal rabbit anti-Ki67 (1/600, Monosan, Uden, The Netherlands) and polyclonal rabbit anti-Iba1 (1/500, Wako Pure Chemical Industries, Osaka, Japan). Antibodyantigen complexes were visualised using a rabbit biotinylated secondary antibody. After incubation with avidin-biotin, the immunoperoxidase staining was visu alised with the Vector NovaRED Substrate Kit (Vector Laboratories Inc., Burlingame, CA, USA). Immunohistochemical detection of the monoclonal mouse antibody against PCNA (1/80, Calbiochem, Germany) was performed using the HistoMouse-MAX kit (Invitrogen Corporation, Camarillo, CA, USA) according to the manufacturer's instructions. Antibody-antigen complexes were visualised using a horseradish peroxidase secondary antibody (Dako North America Inc., Carpinteria, CA, USA) and the DAB chromogen system (Dako North America Inc., Carpinteria, CA, USA).

\section{Visualisation and morphometric analyses}

Thickness of the (neo)cortex: To determine the thickness of the neocortex at $24 \mathrm{~h}$ after irradiation and of the cortex at 1 week after irradiation, the average of 10 random measurements within the region of interest (outlined in Figure 2A,C) was calculated for one section per embryo. The imaging software NIS-Elements BR 4.00.05 (Nikon Instruments Europe B.V., Italy) was used.

Volumetric analysis of the brain: Serial coronal sections were sampled every $100 \mu \mathrm{m}$ through all cerebral hemispheres in embryos at 1 week post irradiation. The area (Figure 2F) was measured in each section, multiplied by $100 \mu \mathrm{m}$ (i.e. the distance between two successive sections) and summed, assuming lack of significant changes between subsequent sections.

Lateral ventricle area analysis: To standardise the sampling, the lateral ventricle area (Figure 2F) was measured in the section with the largest brain area and expressed as the percentage of the whole brain area.

Expression analysis in the embryo: For cleaved caspase3 (CC3), Iba1, PCNA and Sox2 analyses, one section of 
A

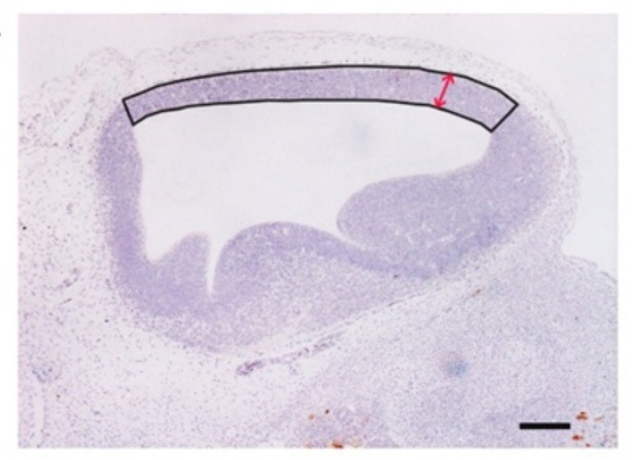

C

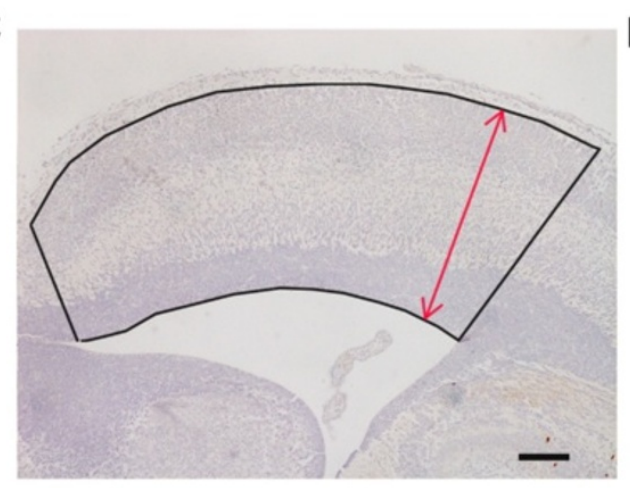

\section{E}

\section{$\mathrm{F}$}

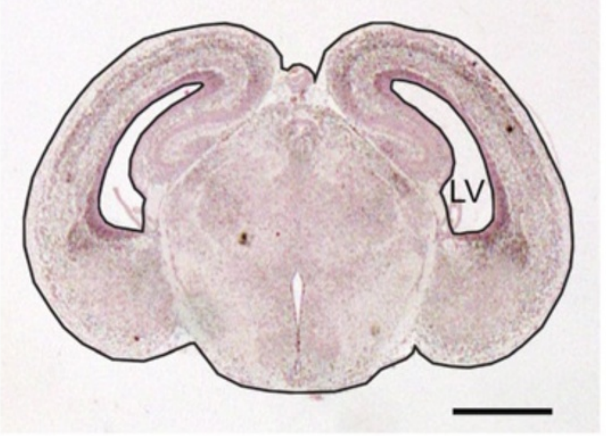

$\mathrm{B}$

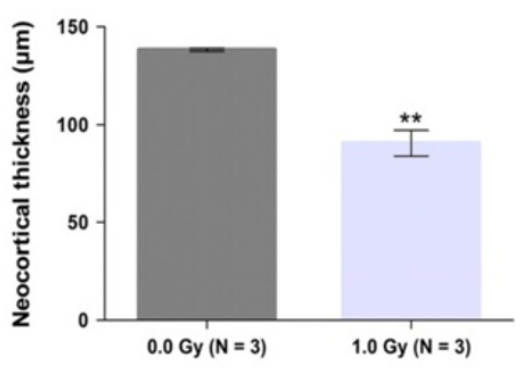

D
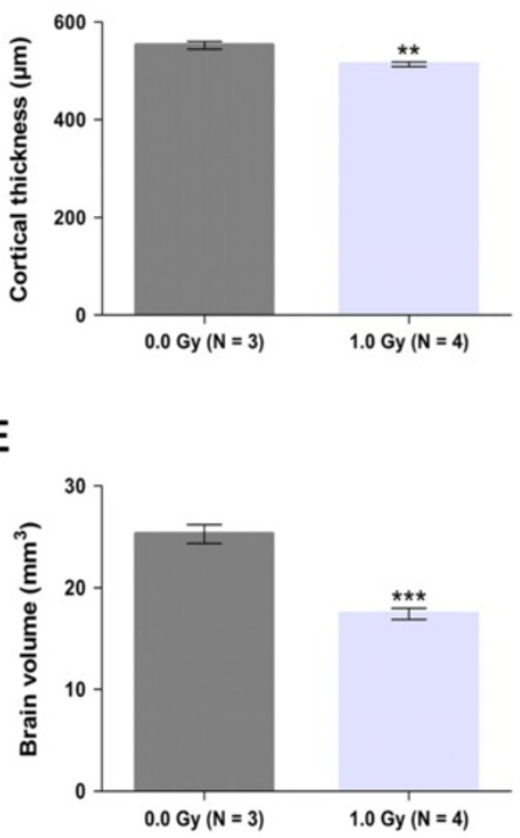

G

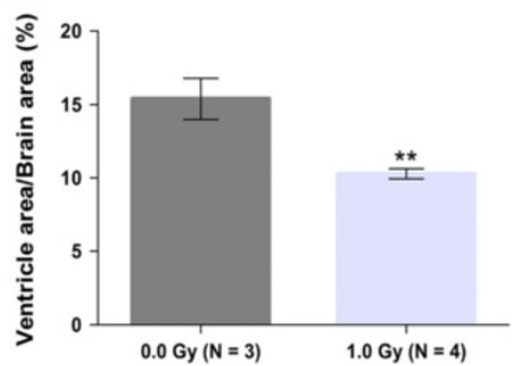

Figure 2 Reduction in brain size, cortical thinning and smaller ventricles in the irradiated embryonic brain. (A, C, F) Representative images are shown for the neocortex at $24 \mathrm{~h}$ after irradiation (A) and for the cortex (C) and the whole brain (F) at 1 week post irradiation with $1.0 \mathrm{~Gy}$, with the indication of the lateral ventricle (LV) and the total brain area (outlined in (F)). (B, D, E, G) Morphometric measurements showed a reduction in neocortical thickness at $24 \mathrm{~h}$ (B) after radiation exposure. Furthermore, at 1 week post irradiation with 1.0 Gy of $\mathrm{X}$-rays, the thickness of the cortex (D), as well as the total brain volume (E) and the ventricle area (G), was significantly reduced in the irradiated embryos. Data are presented as mean \pm SEM. ${ }^{* *} P<0.01,{ }^{* * *} P<0.001$ for comparison with controls. The number of animals used per test is indicated in the graphs $(M)$. Scale bar: $100 \mu \mathrm{m}$ in (A) and (C), 1,000 $\mu \mathrm{m}$ in (F). 
the embryonic brain (at $24 \mathrm{~h}$ or 1 week after irradiation) was immunostained and imaged with HistoFAXS software (TissueGnostics, Austria) at a 10× magnification. Specific regions of interest (delineated in Figure 3A,C) were analysed with HistoQuest software (TissueGnostics, Austria) for automatic colour separation and quantification and staining was expressed as stained positive area per $\mu \mathrm{m}^{2}$.

Analysis of hippocampal neurogenesis: Cell quantification was performed on sagittal sections. The sections were collected starting from $500 \mu \mathrm{m}$ to the midline and two or four non-overlapping sections from each hemisphere, representing the rostral/mid-hippocampus, were taken. For quantification of radial glial cells, GFAP-stained sections were imaged with HistoFAXS at a 10× magnification. The subgranular zone (SGZ) area of the dentate gyrus (DG; dashed line in Figure 4B, upper panel) was analysed with HistoQuest software for automatic colour separation and quantification. All images were analysed using identical software settings. The number of GFAP-positive cells was expressed as the fraction of the labelled area out of the total SGZ area. For Ki67, Sox2 and DCX quantification, the number of positive cells in the SGZ was expressed per DG length $(\mu \mathrm{m})$ (dashed line in Figure 4B, lower panel), measured by the imaging software NIS-Elements BR 4.00.05.

\section{Statistics}

Statistical analyses were performed using GraphPad Prism 5.0. Statistical significance was determined using a two-tailed Student's $t$-test for comparison between pairs of means. $P$ values $<0.05$ were considered to be statistically significant.

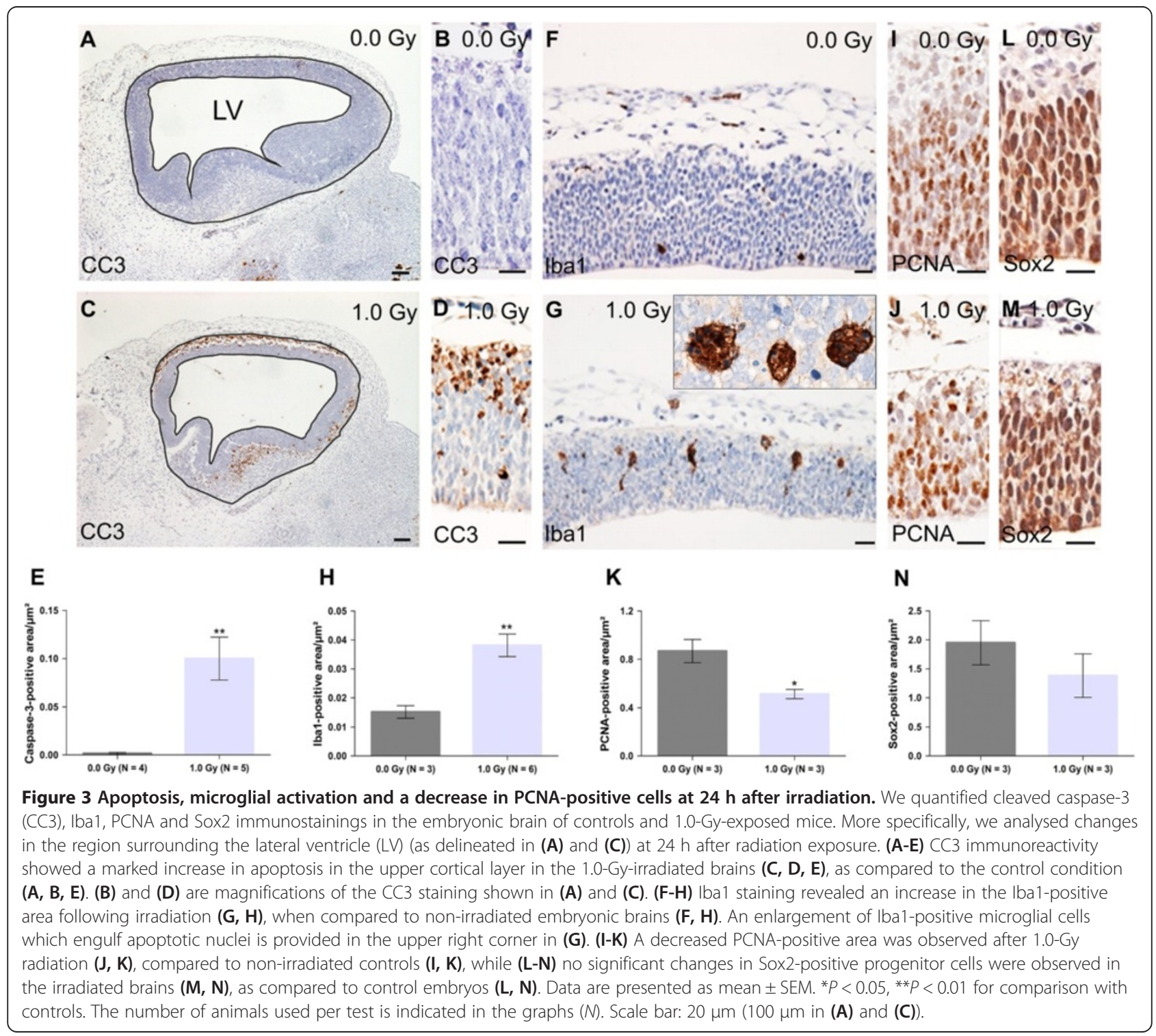




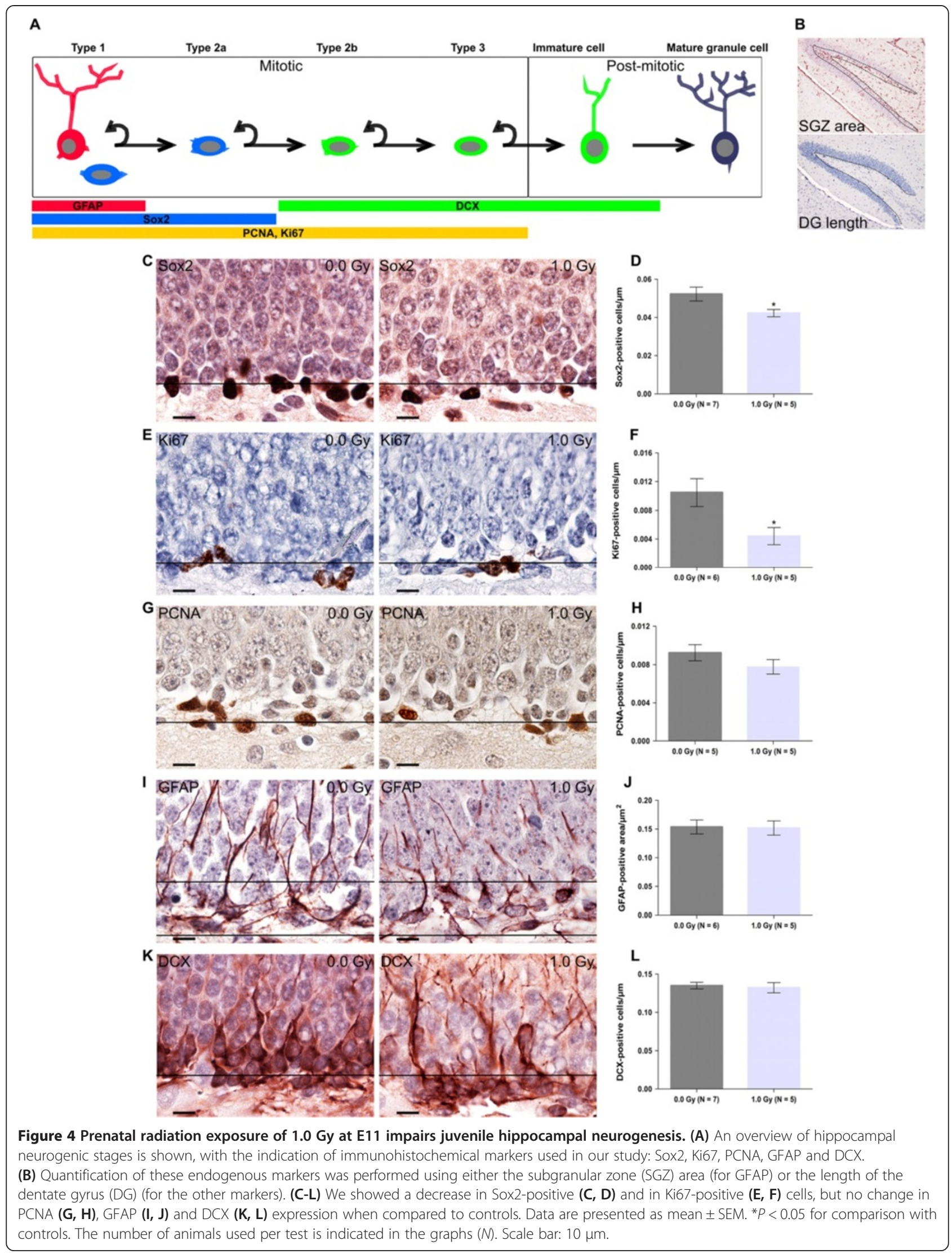




\section{Results}

Exposure to X-rays at the onset of neurogenesis elicits a behavioural response in young adult mice dependent on the timing of irradiation

Male animals irradiated with different doses of X-rays $(0.0,0.2,0.5$ or $1.0 \mathrm{~Gy})$ at selected time points of embryonic development (E10, E11 or E12) were subjected to a series of behavioural tests at the young adult age of 12 weeks. For all experimental groups, we analysed hippocampal-dependent spatial learning and memory in the MWM. Both the acquisition phase, composed of eight daily trial blocks, as well as a probe trial, performed at 4 days after the final acquisition trial block, were analysed. Furthermore, we measured circadian cage activity and examined motor ability using the accelerating rotarod test.

The performance of all experimental groups during the MWM acquisition trials was improved (effect of trial block on escape latency: $P<0.001)$. Irradiation of pregnant mice at E10 did not cause deviations in MWM acquisition when compared to sham-irradiated controls (effect of irradiation dose on escape latency: $P=0.308$, Figure 5A). However, animals irradiated with 0.2 or 1.0 Gy of X-rays did show a lack of preference for the target quadrant during the probe trial, as shown by a similar amount of time spent in the opposite quadrant as in the target quadrant (Figure 5B). This might be suggestive of an impaired consolidation of reference memory without spatial learning defects [31]. Interestingly, spatial learning was affected in animals exposed to radiation at E11. Here, irradiation with 1.0 Gy of X-rays resulted in deviating learning curves during the MWM acquisition as compared to mice exposed to lower doses and controls (effect of irradiation dose on escape latency: $P<0.001$, Figure 5C). Although these mice spent more time in the target quadrant than in the opposite quadrant during the probe trial, the overall swim pattern was very different from that in control animals (Figure 5D). Indeed, animals irradiated with $1.0 \mathrm{~Gy}$ at E11 did not spend significantly more time in the target quadrant versus the adjacent Q2 quadrant while the amount of time spent in the target quadrant was significantly reduced compared to controls. Thus, both spatial learning and memory were affected in these mice. Finally, in mice irradiated at E12, we did not observe differences in MWM acquisition (effect of trial block on escape latency: $P=$ 0.981 , Figure 5E) nor in target quadrant preference (Figure 5F). Finally, control mice of the E11 irradiation group differed mildly in adjacent Q2 and opposite quadrant preference when compared to controls from E10 and E12, possibly attributed to inter-group variability. Yet, no difference in target quadrant preference could be observed, indicating that all mice learned the Morris water maze paradigm and could be used as appropriate controls. In all, spatial learning and long-term memory seem most affected by an irradiation dose of $1.0 \mathrm{~Gy}$ at E11.

In a second test, we analysed 23-h ambulatory horizontal cage activity. When irradiated at E10, no alterations in circadian rhythm were detected between control and irradiated mice (effect of irradiation dose on beam crossings: $P=0.975$, Figure 6A), whereas animals exposed to 0.5 Gy of X-rays at E11 did show mild hyperactivity, mainly observed during the dark phase, in comparison to control animals (effect of irradiation dose on beam crossings: $P=0.013$, Figure $6 \mathrm{~B}$ ). Yet, this hyperactivity was not observed in animals exposed to a higher dose of 1.0 Gy or a lower dose of 0.2 Gy at E11. Finally, there was no significant difference in overall 23-h spontaneous cage activity between mice that had been shamirradiated or X-irradiated at E12 (effect of irradiation dose on beam crossings: $P=0.232$ ). However, at this embryonic age, post hoc testing revealed hyperactivity of all the irradiated groups during the light phase while showing hypoactivity during the dark phase (Figure 6C). Strikingly, when looking into more detail at activity patterns during the dark phase, we could show alterations in nocturnal activity at all three irradiation stages (twoway RM ANOVA, effect of irradiation dose on beam crossings during the night: $P=0.014, P<0.001$ and $P<$ 0.001 for E10, E11 and E12, respectively). Altogether, we found an effect of prenatal irradiation on cage activity patterns, with the most pronounced changes occurring after X-ray exposure at E12.

Lastly, in the rotarod test, we did not observe significant differences in the latency to fall of the rod in mice irradiated at E10 (effect of irradiation dose on latency: $P=0.233$, Figure 6D) or E12 (effect of irradiation dose on latency: $P=0.112$, Figure $6 \mathrm{~F}$ ). On the other hand, mice exposed to $1.0 \mathrm{~Gy}$ of radiation at E11 did show a significant motor defect (effect of irradiation dose on latency: $P=0.006$, Figure $6 \mathrm{E}$ ). Hence, motor coordination and equilibrium was only affected by an irradiation dose of 1.0 Gy at E11, the developmental time point and dose at which irradiation also induced the most deviant spatial learning behaviour.

In conclusion, radiation exposure at the onset of neurogenesis clearly affected long-term neurobehavioural characteristics, which depend on the day of exposure. For further experiments, we aimed at investigating the effects of prenatal irradiation on the brain using a multidisciplinary approach, including MRI and MRS, DTI, gene expression profiling, histology and immunohistochemistry. Because of obvious constraints for such a thorough analysis, we focused only on mice irradiated with 1.0 Gy at E11 for the remainder of the study because these mice showed the clearest defects in behaviour. Moreover, it has previously been shown that the neuronal 


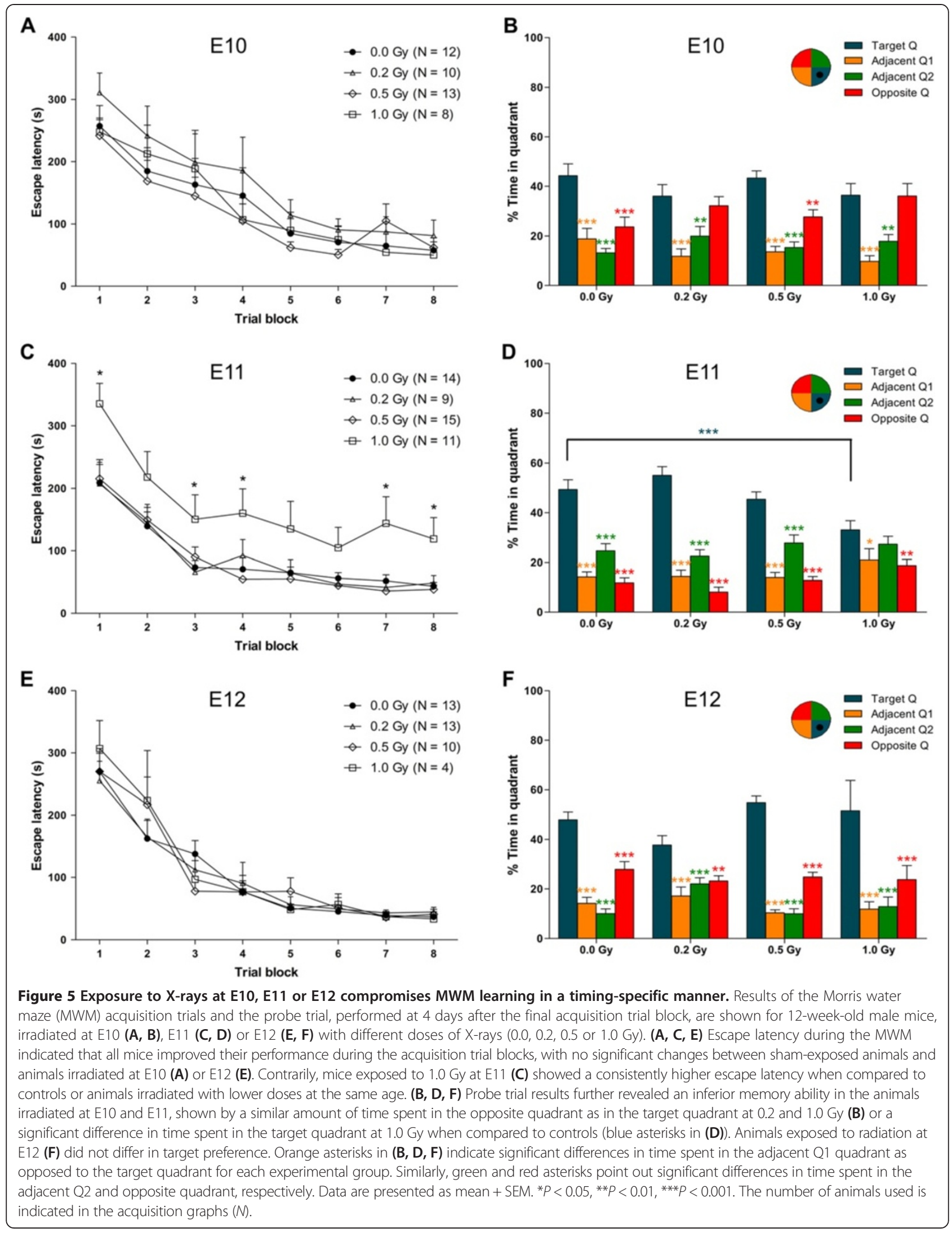




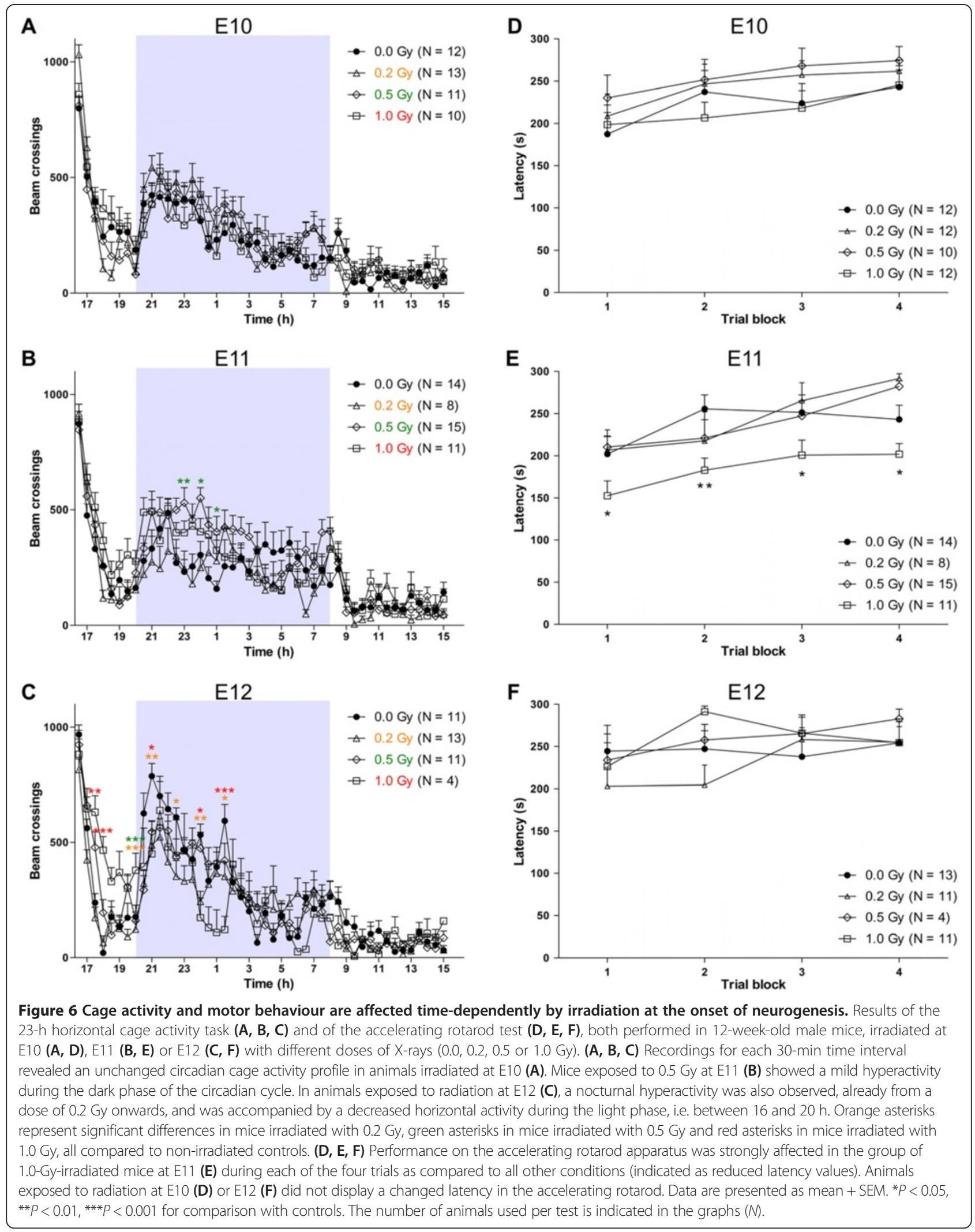


precursor pool at E11 is critical for later brain size [32], a feature which is often affected by prenatal radiation exposure $[33,34]$.

\section{Microstructural alterations in multiple brain regions of animals irradiated at E11}

To investigate possible causes for the observed longterm behavioural effects after prenatal irradiation at E11, we performed in vivo DTI in animals at a young adult (12 weeks old) and adult (40 weeks old) age. The FA of the average water diffusion in DTI represents anisotropy within axons, providing a useful tool to study central nervous system connectivity. In addition, it is highly sensitive to both white and grey matter microstructural changes, stressing its suitability to detect neuropathologies and variations in microstructural composition or tissue architecture [35]. We measured FA (Figure 7A) in defined brain regions, such as the hippocampus, thalamus, sensory-motor cortex and corpus callosum (Additional file 1: Figure S1C). When compared to controls, lower FA values were observed in the hippocampus, thalamus and corpus callosum, but not in the sensory-motor cortex of the irradiated animals at the age of 12 weeks (Figure 7B). In adult mice, reduced FA values were still detected in the hippocampus and corpus callosum, but no longer in the thalamus. Interestingly, at this later stage, FA values in the sensory-motor cortex were now also found to be decreased (Figure 7C).

Altogether, we observed reduced FA in various brain regions of both young adult and adult mice irradiated at E11 with 1.0 Gy, indicative of microstructural changes. A significantly persistent decrease in FA was noted in the hippocampus and corpus callosum. However, since FA is very sensitive, but fairly non-specific [35], other measures are necessary to provide a conclusive cause for the alterations observed.

\section{Irradiation-induced cortical depletion of the neuronal marker NAA}

In the 40-week-old animals, we also calculated metabolite concentrations from localised MR spectra of a cortical and subcortical (striatal) region (Figure 1A,B). This revealed significantly lower concentrations of the nervous system-specific metabolite NAA in the cortex of the irradiated animals (Figure $1 \mathrm{C}$ ), indicative of a pathological loss of neurons [36,37]. None of the other metabolite concentrations examined differed between the two conditions (Additional file 3: Table S2).

In conjunction with the DTI results, we thus observed prenatal radiation-induced defects in both the cerebral

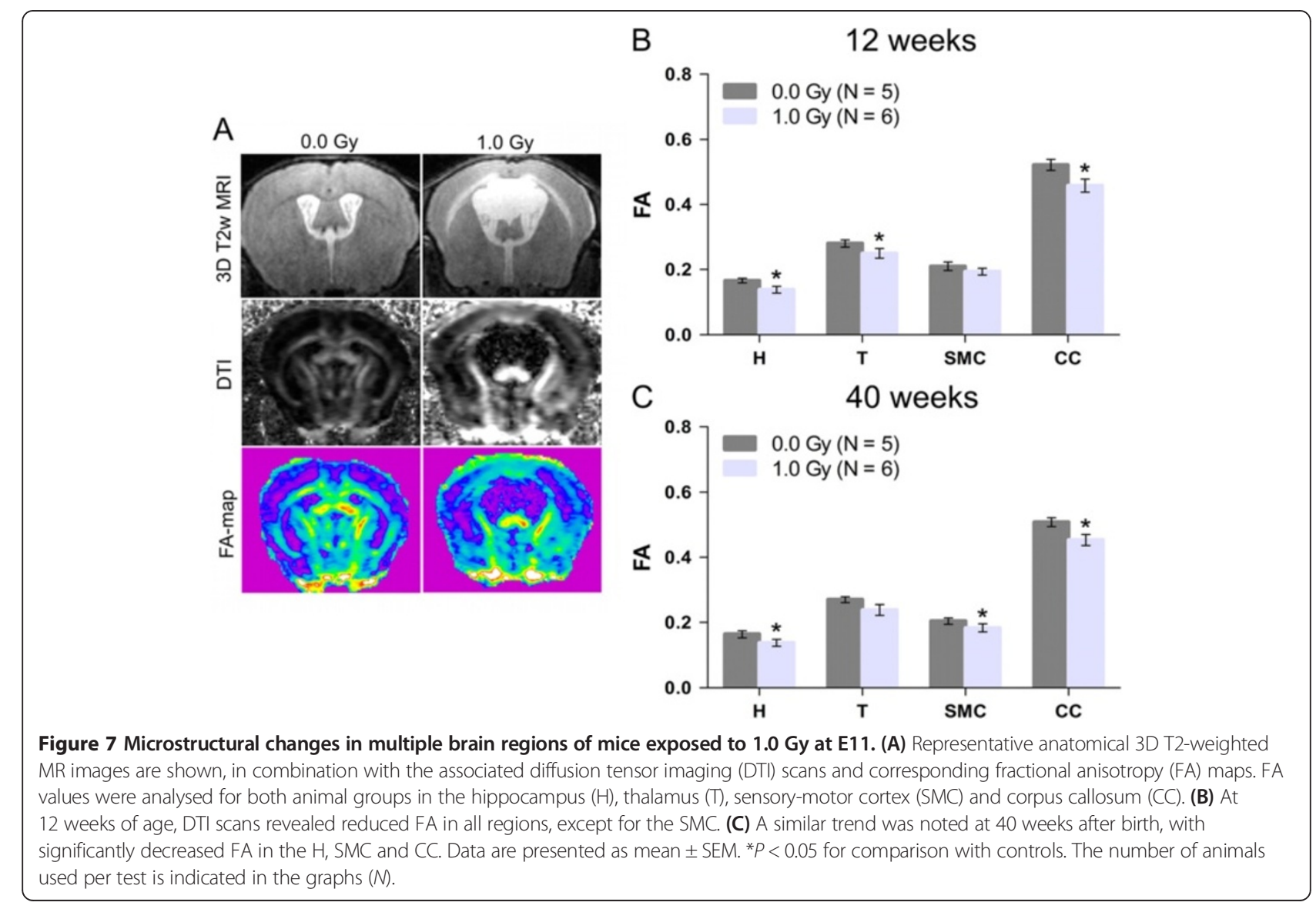


cortex and the hippocampus. For this reason, we focused on those two brain regions for further analyses.

\section{Exposure to radiation at E11 induces a rapid but transient p53-mediated transcriptional response}

The long-term effects of in utero exposure to ionising radiation on the function and structure of the brain are complex and may depend, at least partially, on the early molecular response to stress signals (reactive oxygen species, DNA damage) elicited by irradiation. In order to better understand these early effects, we analysed genomewide changes in gene expression of the whole brain both at 2 and $24 \mathrm{~h}$ after exposure of E11 mouse embryos to different doses of X-rays (0.0, 0.1, 0.2, 0.5 or $1.0 \mathrm{~Gy})$.

For the earliest response, we found 41 genes that were differentially expressed after irradiation, with most genes showing a dose-dependent increase in expression. Functional enrichment analysis showed that these genes are mainly involved in the classical p53-regulated DNA damage response, i.e. genes involved in cell cycle arrest (e.g. Ccng1, Cdkn1a), DNA repair (e.g. Polk, Ercc5) and apoptosis (e.g. Bax, Bbc3, Tnfrsf10b) (Figure 8A). Further evidence for the importance of p53 in mediating these early gene expression changes came from a search in the ChIP enrichment analysis database [29]. Indeed, we found that p53 was by far the most significantly enriched transcription factor predicted to regulate these genes (Figure 8B). Other examples of predicted regulators of the gene expression changes we observed after $2 \mathrm{~h}$ were E2f1, which is known to initiate pro-apoptotic responses to DNA damage [38], and Smad2, which was recently shown to be localised to radiation-induced doublestrand breaks and was proposed to cooperate with p53 to regulate the DNA damage response [39].

At $24 \mathrm{~h}$ after radiation exposure, only three genes (Eda2r, Ccl3 and Nhlrc1) were identified as being differentially expressed in the irradiated embryos, of which the pro-apoptotic p53 target gene Eda2r was also differentially expressed after $2 \mathrm{~h}$. This indicates that the early p53-mediated transcriptional response was only transiently activated after radiation exposure. Interestingly, for Ccl3, known to be involved in inflammation $[40,41]$, we could observe a dose-dependent induction of gene expression, which might be suggestive of a radiation-induced inflammatory response.

To validate some of these results, we performed qRTPCR on a selection of genes. p53 targets (Cdkn1a, Eda2r and Tnfrsfiob) showed a strong and dose-dependent increase after $2 \mathrm{~h}$, which was either attenuated ( $C d k n 1 a$, Eda2r) or completely abrogated (Tnfrsf $10 b$ ) at $24 \mathrm{~h}$ (Figure $8 \mathrm{C}-\mathrm{E}$ ). In contrast, the pro-inflammatory gene $\mathrm{Ccl} 3$ showed a dose-dependent increase only after $24 \mathrm{~h}$ (Figure 8F). Together, our gene expression data thus suggest an early radiation-induced p53-dependent response that might be accompanied or followed by an inflammatory response at $24 \mathrm{~h}$ after irradiation.

\section{Radiation-induced early- and long-term effects: an eye on the cortex}

As previously mentioned, we detected both radiationdependent reduced FA and decreased NAA concentrations in cerebral cortical regions of the adult brain. In order to identify possible causes for these observations, we next focused on the cortical region for more indepth follow-up experiments. To this end, we used immunohistochemistry and morphometric analyses to investigate brain morphology and protein expression at $24 \mathrm{~h}$ and 1 week after irradiation at E11 with 1.0 Gy. Additionally, postnatal brain and cortical morphology were assessed using MRI.

First, to further validate the gene expression results, we analysed apoptosis and microglial inflammation in the embryonic neuroepithelium surrounding the lateral ventricle, encompassing the neocortex (outlined in Figure $3 \mathrm{~A}$ ), using $\mathrm{CC} 3$ and Iba1 immunohistochemistry, respectively. At $24 \mathrm{~h}$ after irradiation, we detected a widespread 64-fold increase in apoptosis in mice irradiated with 1.0 Gy (Figure 3A-E). Remarkably, we observed a strong expression of $\mathrm{CC} 3$ in the outer layers of the neocortex, whereas almost no staining could be observed in the inner ventricular zone. This indicates that apoptosis primarily affected differentiating neurons, rather than progenitor cells. Moreover, the activation of neuroinflammation in the irradiated embryonic brain was confirmed by a clear increase in total Iba1-positive area $24 \mathrm{~h}$ after irradiation (Figure $3 \mathrm{~F}-\mathrm{H}$ ). Visual inspection of the immunostaining showed that there was an increase both in the absolute number and in the size of the Iba1-positive cells in the irradiated embryonic brains compared to controls. Importantly, similar to the localisation of CC3-positive cells and in concordance with the well-recognised phagocytic function of microglia in the brain [42,43], the Iba1 staining was mainly found in the outer layers of the neocortex, where Iba1-positive microglial cells were shown to engulf whole cells (magnification in Figure 3G). Furthermore, progenitor cell proliferation was significantly decreased in the irradiated embryos, as shown by a reduced expression of the cell cycle marker PCNA (Figure 3I-K). In contrast, the amount of Sox2-positive progenitor cells was not affected (Figure 3L-N). Finally, and presumably related to the increased apoptosis and decreased proliferation, the thickness of the neocortex was significantly reduced at $24 \mathrm{~h}$ after radiation exposure in comparison to unexposed controls (Figure 2A,B).

At 1 week post irradiation, apoptosis and proliferation were restored to control levels in the cerebral cortex (Additional file 4: Figure S2), while the difference in 
A

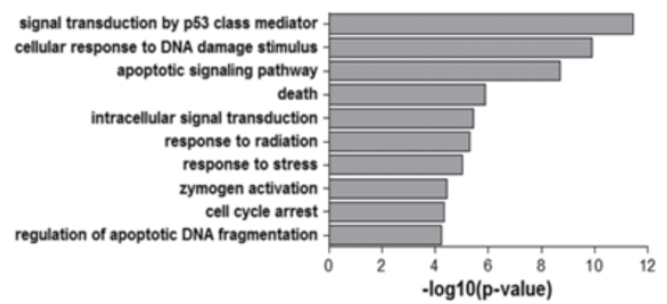

B
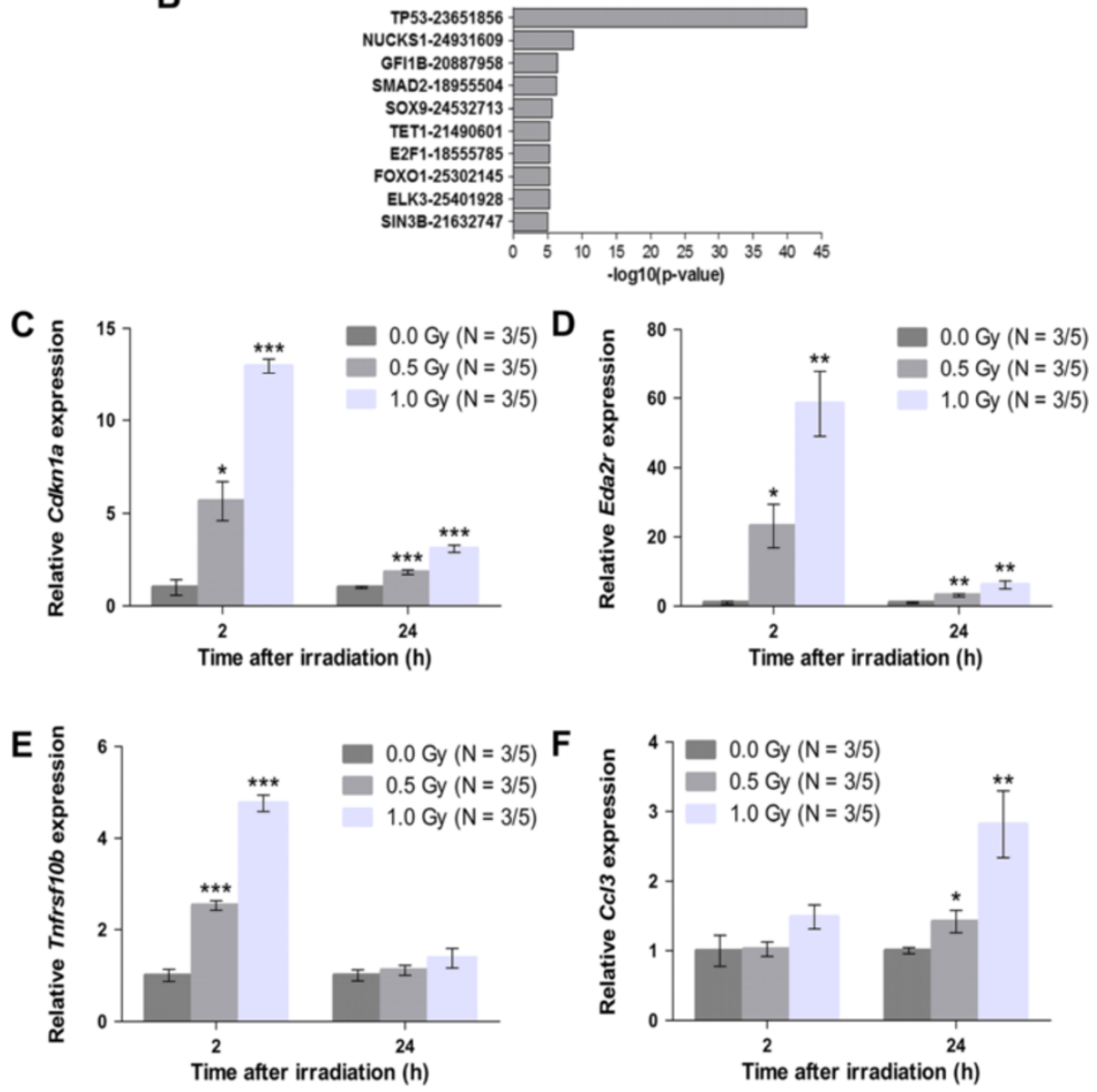

Figure 8 An irradiation-induced early p53-mediated response is associated with the activation of the pro-inflammatory gene Ccl3.

(A, B) Gene Ontology (GO) and transcription factor binding site enrichment among differentially expressed genes in the mouse embryonic brain $2 \mathrm{~h}$ after exposure of the E11 pregnant mother to $1.0 \mathrm{~Gy}$ of X-rays. (A) Top $10 \mathrm{GO}$ enrichment terms among differentially expressed genes at $2 \mathrm{~h}$ after irradiation showed a main involvement of the classical p53-regulated DNA damage response early after irradiation. (B) A chromatin immunoprecipitation (ChIP) enrichment analysis was employed to predict transcriptional regulators of differentially expressed genes $2 \mathrm{~h}$ after irradiation. (C-F) Quantitative reverse transcriptase PCR experiments were applied on a set of genes to confirm microarray findings: p53 targets (Cdkn1a (C), Eda2r (D) and Tnfrsf10b (E)) and CCl3 (F). Data are presented as mean \pm SEM. ${ }^{*} P<0.05$, ${ }^{* *} P<0.01$, ${ }^{* * *} P<0.001$ for comparison with controls. The number of animals used per test is indicated in the graphs (N/N for $2 \mathrm{~h} / 24 \mathrm{~h}$ after irradiation).

cortical thickness was still apparent (Figure 2C,D). Furthermore, the overall brain volume of these irradiated animals was decreased (Figure 2E). Notably, the area of the lateral ventricles, normalised for brain area, was also significantly reduced in the irradiated mice as compared to non-irradiated animals (Figure 2F,G).
To test whether these radiation-induced morphological differences were still evident postnatally, we subjected animals to 3D T2-weighted MRI (Figure 9A). For this purpose, the non-invasive MRI procedure allowed us to image mice at different time points during their lifetime, ranging from postnatal/juvenile ( 1 and 4 weeks 


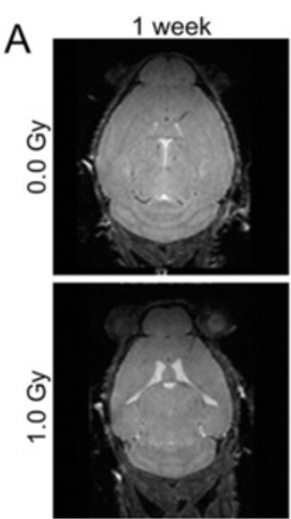

B

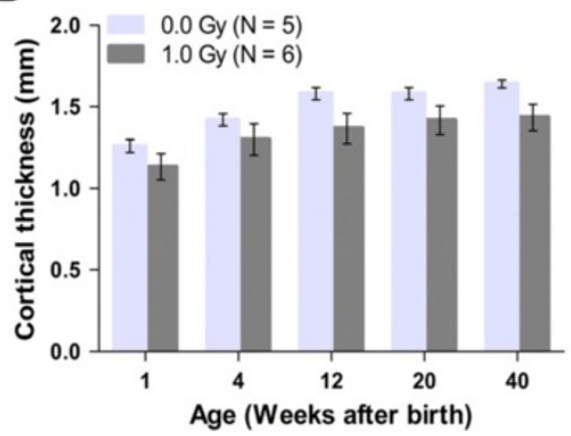

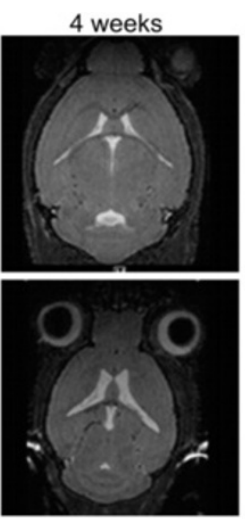

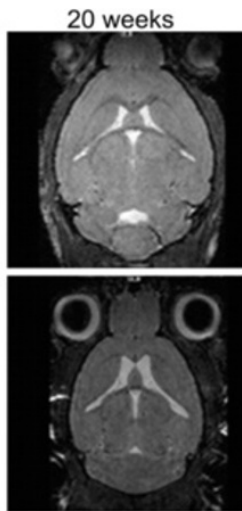

C

c

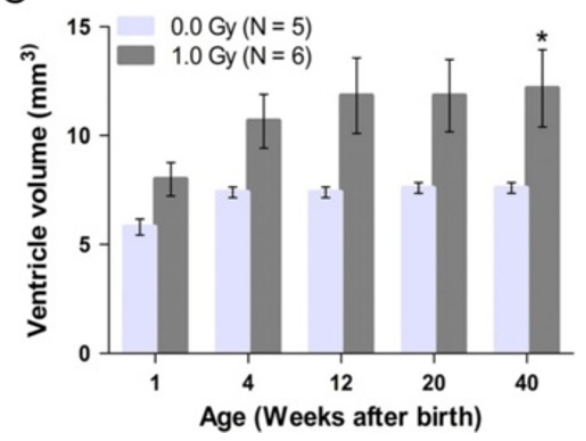

Figure 9 Animals exposed to $\mathbf{1 . 0}$ Gy at E11 display persistent changes in brain anatomy. (A) Representative anatomical 3D T2-weighted MR images of control animals and mice irradiated with $1.0 \mathrm{~Gy}$ at E11 at 1, 4 and 20 weeks after birth. (B) Quantitative analysis of these MR images demonstrated that the cortical thickness of the irradiated mice was mildly decreased, although not statistically significant. (C) At similar time points, these animals showed dilated ventricles, which was significant in 40 -week-old animals. Data are presented as mean \pm SEM. ${ }^{*} P<0.05$ for comparison with controls. The number of animals used per test is indicated in the graphs $(N)$.

after birth) until young adult (12 weeks after birth) and adult (20 and 40 weeks after birth) stages. These sequential analyses demonstrated mild differences in brain anatomy between control and irradiated mice. More specifically, while the irradiated animals did not show a reduction in cortical thickness (Figure 9B), a significant enlargement of the ventricles was noted at 40 weeks of age (Figure 9C). Hence, in the cerebral cortex, early radiation-induced defects may eventually lead to lateoccurring morphological alterations.

\section{Radiation-induced early- and long-term effects: an eye on the hippocampus}

As mentioned before, prenatal irradiation at E11 resulted in defects in spatial learning and memory in the MWM test, suggesting impaired hippocampal function. Since early postnatal radiation exposure has repeatedly been shown to negatively affect hippocampal neurogenesis [44-46], we decided to investigate neurogenesis in the developing and juvenile hippocampus.

At 1 week post irradiation, no difference was found in CC3, PCNA or Sox2 immunoreactivity (Additional file 5: Figure S3). Next, we investigated radiation-induced changes in the cellular composition of the juvenile hippocampal DG. In mice, development of the DG is completed by the fourth postnatal week, the time at which precursors settle in the SGZ and start to generate neurons throughout life, a process referred to as hippocampal neurogenesis [47]. Postnatal hippocampal neurogenesis is a multistep process that recapitulates all stages of neuronal development, however in a mature central nervous system. Type 1 radial stem cells, exhibiting astrocytic properties, express GFAP, whereas Sox2 immunoreactivity can be found, besides in GFAP-positive radial stem cells, in horizontal type 1 stem cells. These stem cells give rise to transient amplifying precursors, of which the type 2a cells still express Sox2 [48]. Upon differentiation and migration to the granule cell layer, immature neurons express DCX [49]. Post-mitotic mature granule neurons further integrate functionally in the DG. We analysed neurogenesis in the DG in 4-week-old mice, corresponding to a juvenile stage characterised by a high rate of neurogenesis [50,51]. Hereto, we employed immunohistochemistry for Sox2, Ki67, PCNA, GFAP and DCX (Figure 4A). Immunoreactivity was assessed by measuring the positively stained area, normalised with respect to the SGZ area for GFAP (upper panel in Figure 4B) or by counting the amount of positive cells, normalised with respect to the length of the 
DG for all other markers (lower panel in Figure 4B). A significant reduction in Sox2-positive cells (Figure 4C,D) and in Ki67 immunoreactivity (Figure 4E,F) was found in the irradiated versus control animals, while the number of PCNA-positive cells (Figure 4G,H), GFAP-positive stem cells (Figure 4I,J) and immature DCX-positive neurons (Figure 4K,L) remained unchanged.

Thus, we can conclude that radiation exposure of the E11 embryo impairs hippocampal neurogenesis through a specific depletion of proliferative and Sox2-positive cells at a juvenile stage. Given that Sox 2 predominantly marks type 1 radial and horizontal stem cells and type 2a intermediate precursors and the fact that no changes were observed in GFAP immunoreactivity, which specifically stains radial stem cells $[48,52]$, we suggest that the horizontal stem cells and/or fast proliferating intermediate cells are suppressed as a result of E11 irradiation.

\section{Discussion}

Several epidemiological studies have provided evidence for neurological aberrations in children prenatally exposed to ionising radiation [4]. For example, in utero radiation exposure after the bombings of Hiroshima and Nagasaki resulted in a higher incidence of small head size, mental retardation, lower IQ values and seizures. Notably, this was only apparent when they had been exposed between gestational weeks 8 and 15 and to a lesser extent between weeks 16 and 25 [6], indicating that the timing of irradiation is very crucial. Nowadays, pregnant women can still be exposed to sources of radiation during medical diagnostic procedures and/or treatment. Radiotherapy, for instance, is sometimes essential to treat cancer patients during pregnancy, but little information exists on the long-term outcome of the exposed embryos or fetuses [9]. Importantly, the time points used in our study are characterised by strongly polarised symmetrical cell divisions in the ventricular zone in order to generate neurons and do not correspond to the radiosensitive gestational period of weeks 8 to 15 in humans [13,53], in which neuronal migration and differentiation primarily occur [10]. This work thus proposes an expansion of the radiosensitivity period, starting even earlier during the course of central nervous system development and resulting in the induction of adult behavioural deficits. A previous study by Baskar and Devi [54] also assessed murine sensitivity to in utero radiation by using behavioural tests in the young adult offspring. Similarly, these authors found a higher susceptibility to ionising radiation during the earliest stages of brain development. However, in their study, the effects were not as distinct between the gestational ages, with only minor differences observed between both mice irradiated at E11.5, E12.5, E14.5 and E17.5. This discrepancy might be explained by the fact that their findings were based on a completely different set of behavioural tests, including the open field for locomotor assessment and the hole board and active avoidance to test learning and memory [54]. Furthermore, in their study, mice of both sexes were anaesthetised in order to irradiate with a higher dose rate than in our experiments. The highest dose used was $0.5 \mathrm{~Gy}$ and experiments were performed in Swiss albino mice, possibly all factors contributing to the non-consistent behavioural results [54]. Here, most obvious behavioural aberrations were noted after irradiation at E11 with a dose of $1.0 \mathrm{~Gy}$, which is why we focused on this condition for further analyses. Intriguingly, the time dependency observed in our study may be due to the disruption of particular cell populations with distinct functions and positioning in the adult brain, which would then also affect different behavioural aspects [19].

In utero exposure to ionising radiation generally induces a classical p53-mediated response, resulting in extensive neuronal cell death, depending on the dose [55-58]. Indeed, we also observed this transient p53dependent apoptotic response after irradiation with a moderate dose of X-rays. Of interest, the CC3 staining pattern at $24 \mathrm{~h}$ after exposure illustrates that most cell death occurs in the outer layers of the neocortex, constituted of differentiating neurons, which is contradictory to previous research in which neural stem or progenitor cells undergo apoptosis in response to irradiation [55]. This finding is also contradictory to one of the dogmas in radiation biology known as the law of Bergonie and Tribondeau, which states that the radiosensitivity of cells is related to their reproductive capacity [59]. Therefore, proliferating cells in the developing brain have always been considered more radiosensitive compared to postmitotic cells [55,60]. Moreover, it has been shown that the DNA damage-induced apoptotic response in differentiating migrating neurons is p21-dependent, whereas stem and progenitor cells repress p21 expression [61]. In accordance with these findings, we also observed a dosedependent increase in the expression of p21 (Cdkn1a) at 2 and $24 \mathrm{~h}$ after irradiation, which may then trigger apoptosis in differentiating neurons.

Irrespective of which cells undergo cell death, the presence of apoptotic cells probably relates to an increase in Iba1-positive microglial cell size at $24 \mathrm{~h}$ after irradiation in the developing neocortex. This, in combination with the appearance of multinucleated microglial cells, indicates phagocytosis of multiple dead cells by activated microglia. Furthermore, we also observed an increase in Iba1 cell number at $24 \mathrm{~h}$ after exposure, indicating inflammatory processes. This was further substantiated by a dose-dependent induction of $\mathrm{Ccl} 3$, a pro-inflammatory cytokine that plays a critical role in radiation-induced inflammation [40,41]. Altogether, these gene expression and immunohistochemical data suggest 
that an inflammatory response to prenatal radiation, involving the activation of microglia, is associated with radiation-induced neuronal cell death. Interestingly, in the prenatally irradiated adult animals, we also found evidence for persistent neuroinflammation and astrogliosis, based on DTI and MRS parameters. The combination of reduced FA and NAA, both apparent in the cortex at 40 weeks of age, is indeed indicative of inflammatory processes and astroglial activation [36,62]. Yet, additional experiments are needed to assess whether this inflammation and/or astrogliosis may contribute to the observed abnormalities.

Further in-depth analysis revealed a reduction in PCNA staining in the area surrounding the lateral ventricle at $24 \mathrm{~h}$ after irradiation. PCNA is highly expressed in dividing cells and its immunoreactivity is detectable in late G1- and S-phase [63]. The reduction in PCNA staining might thus reflect an irradiation-induced block in the cell cycle since gene expression analysis also revealed an upregulation of genes involved in cell cycle arrest.

Radiation-induced apoptosis in the preplate, subsequent microglial cell activation and a decreased proliferation in the ventricular zone of the developing cortex logically result in cell loss, which is in line with the observation of a reduced cortical thickness at $24 \mathrm{~h}$ and 1 week after irradiation. However, as opposed to previous studies demonstrating postnatal thinning of the cortex in prenatally irradiated animals $[34,64]$, we could not demonstrate such a significant difference after birth. Nevertheless, we noted a decline in concentration of cortical NAA, a key MRS marker for vital neuroaxonal tissue, in 40-week-old animals, indicative of a pathological loss of neurons or atrophy [36,37]. Finally, such a decrease in cellular density might also explain the dilation of the ventricles at 40 weeks after birth. In fact, prenatal apoptosis has already been linked to severe postnatal hydrocephalus [65], further substantiating the early- and late-term consequences shown in this study. Still, additional studies will be required to explain the reduction in ventricular size at 1 week post irradiation and a dilation of the ventricles after birth. Possibly, the transient reduction in lateral ventricle size may be a direct consequence of the reduced cell proliferation, considering that cell divisions are required for proper ventricular development [66] and that an interaction exists between the production of embryonic cerebrospinal fluid and proliferation, although mechanisms remain undiscovered [67].

At 4 weeks after birth, in the DG, the level of Sox2positive cells, but not that of GFAP-positive radial stem cells, was significantly reduced in irradiated mice. Analysis of hippocampal proliferation at this juvenile age revealed no changes in PCNA-positive cells. Given that PCNA is only expressed in G1- and S-phase cycling cells [63], Ki67 immunostainings were performed to label dividing cells in all cell cycle phases [52]. This led to the observation of a decreased overall proliferation in the irradiated hippocampus compared to non-irradiated controls. Further investigation is desired to unravel whether the decline in proliferation and in Sox2-positive stem cells, by depleting the stem cell pool, has consequences for later maturation and synaptic functional integration of these neurons. For instance, immunohistochemistry for synaptic markers and electrophysiological recordings might be of great interest considering that an impairment of hippocampal neurogenesis is strongly linked to cognitive defects [68]. To our knowledge, only one study has previously analysed radiation-induced effects on hippocampal neurogenesis, by using BrdU labelling of $\mathrm{S}$-phase cells in the hippocampus of 12-week-old animals. These authors did not find a difference in neurogenesis between non-irradiated and 1.0-Gy-irradiated E16 rats, although MWM screening showed a deficiency in shortterm, but not long-term, spatial memory [69]. Notably, and opposed to the study of Tomasova et al., we analysed neurogenesis in juvenile mice, of which the observed neurogenic decrease might have more serious consequences for brain structure and cognition than a decrease in adult neurogenesis. Indeed, juvenile neurogenesis was proposed to be indispensable in the formation of $25 \%$ of the total number of hippocampal granule neurons. Thereafter, in young adult and adult brains, the granule neuronal number remains stable and neurogenesis is only deemed to be necessary for cell replacement [70].

Previous research indicated that behavioural defects after in utero radiation exposure highly depend upon the evident morphological changes, such as brain volume decrease, ventricular enlargement and cortical thinning [71]. This is further evidenced in non-human primates, in which morphological effects induced by prenatal radiation exposure are thought to be responsible for neurological symptoms [72,73]. Here, we observed both abnormalities in neurogenesis as well as morphological aberrations. Therefore, we cannot conclusively dissect the exact cause for inducing the cognitive defects. Moreover, as both alterations occur in different brain regions, an interplay and interconnectivity between distinct neuronal cell types may be responsible for the observed behavioural phenotype. For this reason, more behavioural tests addressing cortical versus hippocampal functions are recommended to segregate the functional outcome of an affected cortical and/or hippocampal development.

\section{Conclusions}

In the present study, we used a multidisciplinary approach combining behavioural assessment, MRI, DTI, MRS, gene expression profiling and immunohistochemistry in order to reveal short- and long-term biological and molecular effects of radiation exposure during the 
early stages of mouse brain development. The observed long-term behavioural deficits and brain structure anomalies likely reflect the developmental defects initiated by radiation-induced DNA damage and subsequent apoptosis and inflammation. However, we could not provide a causal link between the early irradiation-induced apoptosis in the embryonic brain and adult behavioural and structural effects. Therefore, to fully correlate the adult phenotypic effects with prenatal apoptosis, experiments abrogating the early p53-dependent apoptotic response would be of use. In any case, we believe our study contributed to a better understanding of the timing and mechanisms responsible for radiation-induced neuronal non-cancer effects occurring several years after exposure. It also further highlights the importance and necessity of radioprotection during pregnancy, in particular during this critical time window of early neurogenesis.

\section{Additional files}

Additional file 1: Figure S1. Illustration of region selection for repeated quantification of MR imaging data in individual animals. (A) The cortical thickness was determined as the distance between the corpus callosum and the boundary of the brain in the axial view. To make the selection of the same location reproducible, we chose the slice where the lateral ventricles and the dorsal third ventricle apparently meet (left panel). Assessment of the cortical thickness up to $0.8 \mathrm{~mm}$ anterior or posterior to this location resulted in differences of the cortical thickness not larger than 2\%-5\% (right panel). (B) Illustration of the determination of the ventricle volumes using the 'region-of-interest' tool of ParaVision 5.1. Initial seeding points were set in the hyperintense regions of the ventricles. Lower and upper intensity thresholds were selected and the 'volume growth' algorithm was chosen for delineation of the ventricles. The selected volumes were then manually corrected. (C) Volumes for the determination of fractional anisotropy (FA) values were selected on 3D T2-weighted MR images. Those volumes were then transferred to the respective FA maps. Values were determined from regions in the corpus callosum (1), motor cortex (2), hippocampus (3) and thalamus (4).

Additional file 2: Table S1. Primer sequences for quantitative reverse transcriptase PCR (qRT-PCR). Primers used for quantitative reverse transcriptase PCR qRT-PCR experiments included: $\mathrm{Cdkn}_{1} a, \mathrm{CCl} 3, \mathrm{Eda} 2 \mathrm{r}$ and Tnfrsfiob. Gapdh was used as a control.

Additional file 3: Table S2. Quantification of metabolites based on localised MR spectroscopy. Single-voxel MR spectra from a cortical and subcortical (striatal) region were acquired for control and E11-irradiated animals at 40 weeks after birth. Quantification was performed relative to the non-suppressed water signal (expressed in $\mu \mathrm{mol} \mathrm{g}^{-1}$ ). Data are presented as mean $\pm S E M$. The number of animals used is indicated.

Additional file 4: Figure S2. No change in cortical apoptosis and proliferation at 1 week post irradiation with $1.0 \mathrm{~Gy}$. We analysed cleaved caspase-3 (CC3) (A) and PCNA (B) in brain sections from embryos irradiated with 1.0 Gy at E11, which showed no marked changes in expression when compared to the sham-irradiated animals. Data are presented as mean \pm SEM. The number of animals used per test is indicated in the graphs $(M)$.

Additional file 5: Figure S3. No difference in hippocampal apoptosis and proliferation at 1 week after E11 irradiation. Cleaved caspase-3 (CC3) (A), PCNA (B) and Sox2 (C) immunoreactivity was quantified in sections of the embryonic hippocampus at 1 week post E11 irradiation with 1.0 Gy. This showed no alterations in expression between irradiated and sham-irradiated mice. Data are presented as mean \pm SEM. The number of animals used per test is indicated in the graphs $(N)$.

\section{Abbreviations}

CC: Corpus callosum; CC3: Cleaved caspase-3; ChIP: Chromatin immunoprecipitation; Chol: Choline; Cre: Creatine; DTI: Diffusion tensor imaging; E: Embryonic day; EPI: Echo planar imaging; FA: Fractional anisotropy; FOV: Field of view; GO: Gene Ontology;

GPC: Glycerophosphorylcholine; H: Hippocampus; LV: Lateral ventricles; MRI: Magnetic resonance imaging; MRS: Magnetic resonance spectroscopy; MWM: Morris water maze; NAA: N-acetyl aspartate; PChol: Phosphorylcholine; PCr: Phosphocreatine; qRT-PCR: Quantitative reverse transcriptase PCR; RARE: Rapid acquisition with refocused echoes; SGZ: Subgranular zone; SMC: Sensory-motor cortex; T: Thalamus; TE: Echo time; TR: Repetition time.

\section{Competing interests}

The authors declare that they have no competing interests.

\section{Authors' contributions}

$M N, L L, A M$ and $A J$ were responsible for the mouse breeding and coupling and performed the gene expression analysis experiments. EDA and GW performed the radiation and dosimetry research and analysed the data. MT, AC and SP carried out the immunohistochemical stainings and morphometric analyses. SB, LM, AS and PPDD participated in the design of the study. DVD performed the behavioural tests and analysed the data. UH participated in the design of the study and analysed the MR data. MAB designed the research and revised the paper. RQ designed the research, analysed the data and revised the paper. TV and MV wrote the paper, and TV analysed the data and performed the statistical analyses. All authors read and approved the final manuscript.

\section{Acknowledgements}

This work was supported by the FP7 Euratom EU project CEREBRAD (Cognitive and Cerebrovascular Effects Induced by Low Dose Ionising Radiation, grant agreement number 295552), the Research Foundation Flanders (FWO - Flanders), the Interuniversity Poles of Attraction of the Belgian Federal Science Policy Office (P7/16), a Methusalem excellence grant of the Flemish Government, an agreement between the Institute Born-Bunge and the University of Antwerp, the Medical Research Foundation Antwerp, Neurosearch Antwerp and the Thomas Riellaerts Research Fund. TV is funded by a joint doctoral SCK-CEN/KU Leuven grant, and DVD is a postdoctoral fellow of the FWO - Flanders. The authors wish to thank Dr. Pieter Monsieurs for the statistical reviewing.

\section{Author details}

${ }^{1}$ Radiobiology Unit, Laboratory of Molecular and Cellular Biology, Institute for Environment, Health and Safety, Belgian Nuclear Research Centre, SCK.CEN, $2400 \mathrm{Mol}$, Belgium. '2Laboratory of Neural Circuit Development and Regeneration, Department of Biology, Faculty of Science, University of Leuven, 3000 Leuven, Belgium. ${ }^{3}$ Laboratory of Neurochemistry and Behaviour, Institute Born-Bunge, Department of Biomedical Sciences, University of Antwerp, 2610 Wilrijk, Belgium. ${ }^{4}$ Laboratory of Radiation Biology and Biomedicine, Agenzia Nazionale per le Nuove Tecnologie, l'Energia e lo Sviluppo Economico Sostenibile (ENEA), Casaccia Research Centre, 00123 Rome, Italy. ${ }^{5}$ SB Dosimetry and Calibration, Institute for Environment, Health and Safety, Belgian Nuclear Research Centre, SCK.CEN, 2400 Mol, Belgium. ${ }^{6}$ Biomedical NMR Unit, Department of Imaging and Pathology, Faculty of Medicine, University of Leuven, 3000 Leuven, Belgium. ${ }^{7}$ Molecular Small Animal Imaging Center (MoSAIC), Faculty of Medicine, University of Leuven, 3000 Leuven, Belgium. ${ }^{8}$ Department of Neurology and Alzheimer Research Center, University of Groningen, University Medical Center Groningen, 9713 GZ Groningen, The Netherlands.

Received: 22 July 2014 Accepted: 22 December 2014

Published: 9 January 2015

\section{References}

1. Rodier PM. Environmental causes of central nervous system maldevelopment. Pediatrics. 2004;113:1076-83.

2. Rice D, Barone JS. Critical periods of vulnerability for the developing nervous system: evidence from human and animal models. Environ Health Perspect. 2000;108:511-33.

3. Diav-Citrin O. Prenatal exposures associated with neurodevelopmental delay and disabilities. Dev Disabil Res Rev. 2011;17:71-84. 
4. Verheyde J, Benotmane MA. Unraveling the fundamental molecular mechanisms of morphological and cognitive defects in the irradiated brain. Brain Res Rev. 2007;53:312-20.

5. Otake M, Schull WJ. Review: Radiation-related brain damage and growth retardation among the prenatally exposed atomic bomb survivors. Int J Radiat Biol. 1998;74:159-71.

6. Schull WJ, Norton S, Jensh RP. lonizing radiation and the developing brain. Neurotoxicol Teratol. 1990;12:249-60.

7. Di Leo M, Arcidiacono PG. Fetal radiation exposure: is monitoring really needed? World J Gastrointest Endosc. 2013;5:366-8.

8. Kal HB, Struikmans H. Radiotherapy during pregnancy: fact and fiction. Lancet Oncol. 2005;6:328-33.

9. Van Calsteren K, Amant F. Cancer during pregnancy. Acta Obstet Gynecol Scand. 2014;93:443-6.

10. Ronan JL, Wu W, Crabtree GR. From neural development to cognition: unexpected roles for chromatin. Nat Rev Genet. 2013;14:347-59.

11. Samuelsen GB, Larsen KB, Bogdanovic N, Laursen H, Graem N, Larsen JF, et al. The changing number of cells in the human fetal forebrain and its subdivisions: a stereological analysis. Cereb Cortex. 2003;13(2):115-22.

12. Pakkenberg B, Gundersen HJ. Neocortical neuron number in humans: effect of sex and age. J Comp Neurol. 1997;384(2):312-20.

13. Workman AD, Charvet CJ, Clancy B, Darlington RB, Finlay BL. Modeling transformations of neurodevelopmental sequences across mammalian species. J Neurosci. 2013;33(17):7368-83.

14. Hatten ME. Central nervous system neuronal migration. Annu Rev Neurosci. 1999;22:511-39.

15. Kappen C. Modeling anterior development in mice: diet as modulator of risk for neural tube defects. Am J Med Genet C: Semin Med Genet. 2013;163C(4):333-56.

16. Schmidt SL, Lent R. Effects of prenatal irradiation on the development of cerebral cortex and corpus callosum of the mouse. J Comp Neurol. 1987;264(2):193-204.

17. De Santis M, Cesari E, Nobili E, Straface G, Cavaliere AF, Caruso A. Radiation effects on development. Birth Defects Res Part C-Embryo Today-Rev. 2007;81:177-82.

18. Friedman HR, Selemon LD. Fetal irradiation interferes with adult cognition in the nonhuman primate. Biol Psychiatry. 2010;68(1):108-11.

19. Algan $O$, Rakic P. Radiation-induced, lamina-specific deletion of neurons in the primate visual cortex. J Comp Neurol. 1997;381(3):335-52.

20. Easter Jr SS, Ross LS, Frankfurter A. Initial tract formation in the mouse brain. J Neurosci. 1993;13(1):285-99.

21. Franklin KBJ, Paxinos G. A stereotaxic atlas of the mouse brain. San Diego: Academic; 1997

22. Bai J, Trinh TL, Chuang KH, Qiu A. Atlas-based automatic mouse brain image segmentation revisited: model complexity vs. image registration. Magn Reson Imaging. 2012;30(6):789-98.

23. Osorio-Garcia MI, Sima DM, Nielsen FU, Dresselaers T, Van Leuven F, Himmelreich $U$, et al. Quantification of in vivo $1 \mathrm{H}$ magnetic resonance spectroscopy signals with baseline and lineshape estimation. Meas Sci Technol. 2011;22:114011.

24. Osorio-Garcia MI, Sima DM, Nielsen FU, Himmelreich U, Van Huffel S. Quantification of magnetic resonance spectroscopy signals with lineshape estimation. J Chemometrics. 2011;25:183-92.

25. Poullet JB, Sima DM, Simonetti AW, De Neuter B, Vanhamme L, Lemmerling $P$, et al. An automated quantitation of short echo time MRS spectra in an open source software environment: AQSES. NMR Biomed. 2007;20:493-504.

26. Benjamini $Y$, Hochberg Y. Controlling the false discovery rate: a practical and powerful approach to multiple testing. JSTOR. 1995;27(1):289-300.

27. Eden E, Navon R, Steinfield I, Lipson D, Yakhini Z. GOrilla: a tool for discovery and visualization of enriched GO terms in ranked gene lists. BMC Bioinformatics. 2009;10:48.

28. Supek F, Bosnjak M, Skunca N, Smuc T. REVIGO summarizes and visualizes long lists of gene ontology terms. Plos One. 2011;6(7):e21800.

29. Lachmann A, Xu H, Krishnan J, Berger SI, Mazloom AR, Ma'ayan A. ChEA transcription factor regulation inferred from integrating genome-wide ChIP-X experiments. Bioinformatics. 2010;26:2438-44.

30. Pfaffl MW. A new mathematical model for relative quantification in real-time RT-PCR. Nucleic Acids Res. 2001;29:e45.

31. Vorhees CV, Williams MT. Morris water maze: procedures for assessing spatial and related forms of learning and memory. Nat Protoc. 2006;1(2):848-58.
32. Gilmore EC, Walsh CA. Genetic causes of microcephaly and lessons for neuronal development. Wiley Interdiscip Rev Dev Biol. 2013;2(4):461-78.

33. Sun $X$, Inouye M, Takagishi $Y$, Hayasaka S, Yamamura H. Follow-up study on histogenesis of microcephaly associated with ectopic gray matter induced by prenatal gamma-irradiation in the mouse. J Neuropathol Exp Neurol. 1996:55:357-65.

34. Saito S, Sawada K, Hirose M, Mori Y, Yoshioka Y, Murase K. Diffusion tensor imaging of brain abnormalities induced by prenatal exposure to radiation in rodents. PLoS One. 2014;9(9):e107368.

35. Alexander AL, Lee JE, Lazar M, Field AS. Diffusion tensor imaging of the brain. Neurotherapeutics. 2007;4(3):316-29.

36. Michaelis T, Boretius S, Frahm J. Localized proton MRS of animal brain in vivo: models of human disorders. Prog Nucl Magn Reson Spectrosc 2009:55:1-34.

37. Moffett JR, Ross B, Arun P, Madhavarao CN, Namboodiri AM. NAcetylaspartate in the CNS: from neurodiagnostics to neurobiology. Prog Neurobiol. 2007:81:89-131.

38. Udayakumar T, Shareef MM, Diaz DA, Ahmed MM, Pollack A. The E2F1/Rb and p53/MDM2 pathways in DNA repair and apoptosis: understanding the crosstalk to develop novel strategies for prostate cancer radiotherapy. Semin Radiat Oncol. 2010;20:258-66.

39. Wang M, Saha J, Hada M, Anderson JA, Pluth JM, O'Neill P, et al. Novel Smad proteins localize to IR-induced double-strand breaks: interplay between TGFB and ATM pathways. Nucleic Acids Res. 2013;41:933-42.

40. Kalm M, Fukuda A, Fukuda H, Öhrfelt A, Lannering B, Björk-Eriksson T, et al. Transient inflammation in neurogenic regions after irradiation of the developing brain. Radiat Res. 2009;171:66-76.

41. Kalm M, Roughton K, Blomgren K. Lipopolysaccharide sensitized male and female juvenile brains to ionizing radiation. Cell Death Dis. 2013;4:e962.

42. Neher JJ, Neniskyte U, Zhao JW, Bal-Price A, Tolkovsky AM, Brown GC. Inhibition of microglial phagocytosis is sufficient to prevent inflammatory neuronal death. J Immunol. 2011:186:4973-83.

43. Fuhrmann M, Bittner T, Jung CK, Burgold S, Page RM, Mitteregger G, et al. Microglial Cx3cr1 knockout prevents neuron loss in a mouse model of Alzheimer's disease. Nat Neurosci. 2010;13:411-3.

44. Fukuda H, Fukuda A, Zhu C, Korhonen L, Swanpalmer J, Hertzman S, et al. Irradiation-induced progenitor cell death in the developing brain is resistant to erythropoietin treatment and caspase inhibition. Cell Death Differ. 2004;11(11):1166-78.

45. Naylor AS, Bull C, Nilsson MK, Zhu C, Bjork-Eriksson T, Eriksson PS, et al. Voluntary running rescues adult hippocampal neurogenesis after irradiation of the young mouse brain. Proc Natl Acad Sci U S A. 2008;105(38):14632-7.

46. Monje ML, Mizumatsu S, Fike JR, Palmer TD. Irradiation induces neural precursor-cell dysfunction. Nat Med. 2002;8(9):955-62.

47. Pedroni A, Minh Do D, Mallamaci A, Cherubini E. Electrophysiological characterization of granule cells in the dentate gyrus immediately after birth. Front Cell Neurosci. 2014;8:44.

48. Hodge RD, Hevner RF. Expression and actions of transcription factors in adult hippocampal neurogenesis. Dev Neurobiol. 2011;71(8):680-9.

49. Kempermann G, Jessberger S, Steiner B, Kronenberg G. Milestones of neuronal development in the adult hippocampus. Trends Neurosci. 2004;27(8):447-52.

50. Gil-Mohapel J, Brocardo PS, Choquette W, Gothard R, Simpson JM, Christie BR. Hippocampal neurogenesis levels predict WATERMAZE search strategies in the aging brain. PLoS One. 2013;8(9):e75125.

51. Kuhn HG, Dickinson-Anson H, Gage FH. Neurogenesis in the dentate gyrus of the adult rat: age-related decrease of neuronal progenitor proliferation. J Neurosci. 1996;16(6):2027-33.

52. Von B, Halbach O. Immunohistological markers for proliferative events, gliogenesis, and neurogenesis within the adult hippocampus. Cell Tissue Res. 2011;345(1):1-19.

53. Okano H, Temple S. Cell types to order: temporal specification of CNS stem cells. Curr Opin Neurobiol. 2009;19(2):112-9.

54. Baskar R, Uma DP. Influence of gestational age to low-level gamma irradiation on postnatal behavior in mice. Neurotoxicol Teratol. 2000;22:593-602.

55. Nowak E, Etienne O, Millet P, Silva Lages C, Mathieu C, Mouthon M-A, et al. Radiation-induced H2AX phosphorylation and neural precursor apoptosis in the developing brain of mice. Radiat Res. 2006;165:155-64.

56. Samari N, De Saint-Georges L, Pani G, Baatout S, Leyns L, Benotmane MA. Non-conventional apoptotic response to ionising radiation mediated by $\mathrm{N}$-methyl D-aspartate receptors in immature neuronal cells. Int J Mol Med. 2013;31:516-24 
57. Verheyde J, de Saint-Georges L, Leyns L, Benotmane MA. The role of Trp53 in the transcriptional response to ionizing radiation in the developing brain. DNA Res. 2006;13:65-75.

58. Semont A, Nowak EB, Silva Lages C, Mathieu C, Mouthon MA, May E, et al. Involvement of p53 and Fas/CD95 in murine neural progenitor cell response to ionizing irradiation. Oncogene. 2004;23:8497-508.

59. Bergonie J, Tribondeau L. De quelques resultats de la radiotherapie et essai de fixation d'une technique rationelle. C R Acad Sci. 1906;143:983-5.

60. Saha S, Woodbine L, Haines J, Coster M, Ricket N, Barazzuol L, et al. Increased apoptosis and DNA double-strand breaks in the embryonic mouse brain in response to very low-dose X-rays but not $50 \mathrm{~Hz}$ magnetic fields. J R Soc Interface. 2014;11(100):20140783.

61. Roque T, Haton C, Etienne O, Chicheportiche A, Rousseau L, Martin L, et al. Lack of a p21 $1^{\text {wafl/Cip }}$-dependent $\mathrm{G} 1 / \mathrm{S}$ checkpoint in neural stem and progenitor cells after DNA damage in vivo. Stem Cells. 2012;30:537-47.

62. Gupta M, Rana P, Trivedi R, Kumar BS, Khan AR, Soni R, et al. Comparative evaluation of brain neurometabolites and DTI indices following whole body and cranial irradiation: a magnetic resonance imaging and spectroscopy study. NMR Biomed. 2013;26(12):1733-41.

63. Kohler T, Prols F, Brand-Saberi B. PCNA in situ hybridization: a novel and reliable tool for detection of dynamic changes in proliferative activity. Histochem Cell Biol. 2005;123:315-27.

64. Miki T, Fukui Y, Takeuchi Y, Itoh M. A quantitative study of the effects of prenatal X-irradiation on the development of cerebral cortex in rats. Neurosci Res. 1995;23(3):241-7.

65. Klezovitch O, Fernandez TE, Tapscott SJ, Vasioukhin V. Loss of cell polarity causes severe brain dysplasia in Lgl1 knockout mice. Genes Dev. 2004;18 (5):559-71.

66. Lowery LA, Sive H. Initial formation of zebrafish brain ventricles occurs independently of circulation and requires the nagie oko and snakehead/ atp1a1a.1 gene products. Development. 2005;132(9):2057-67.

67. Lowery $L A$, Sive $H$. Totally tubular: the mystery behind function and origin of the brain ventricular system. Bioessays. 2009;31(4):446-58.

68. Garthe A, Kempermann G. An old test for new neurons: refining the Morris water maze to study the functional relevance of adult hippocampal neurogenesis. Front Neurosci. 2013;7:63.

69. Tomasova L, Smajda B, Sevc J. Effects of prenatal irradiation on behaviour and hippocampal neurogenesis in adult rats. Acta Physiol Hung. 2012;99:126-32.

70. Cushman JD, Maldonado J, Kwon EE, Garcia AD, Fan G, Imura T, et al. Juvenile neurogenesis makes essential contributions to adult brain structure and plays a sex-dependent role in fear memories. Front Behav Neurosci. 2012;6:3.

71. Kimler BF. Prenatal irradiation: a major concern for the developing brain. Int J Radiat Biol. 1998;73:423-34.

72. Selemon LD, Ceritoglu C, Ratnanather JT, Wang L, Harms MP, Aldridge K, et al. Distinct abnormalities of the primate prefrontal cortex caused by ionizing radiation in early or midgestation. J Comp Neurol. 2013;521(5):1040-53.

73. Selemon LD, Friedman HR. Motor stereotypies and cognitive perseveration in non-human primates exposed to early gestational irradiation. Neuroscience. 2013;248C:213-24.

doi:10.1186/1866-1955-7-3

Cite this article as: Verreet et al: A multidisciplinary approach unravels early and persistent effects of $X$-ray exposure at the onset of prenatal neurogenesis. Journal of Neurodevelopmental Disorders 2015 7:3.

\section{Submit your next manuscript to BioMed Central and take full advantage of:}

- Convenient online submission

- Thorough peer review

- No space constraints or color figure charges

- Immediate publication on acceptance

- Inclusion in PubMed, CAS, Scopus and Google Scholar

- Research which is freely available for redistribution 